\title{
Kelvin's Dissymmetric Models and Consistency Conditions of Multicomponent Gas-Liquid Equilibrium and Capillary Condensation
}

\author{
Mikhail Panfilov ${ }^{1}$ and Alexandre Koldoba ${ }^{2}$ \\ ${ }^{1}$ Laboratoire d'Energétique et de Mécanique Théorique et Appliquée, CNRS-UMR 7563, Université de Lorraine, 54500 Nancy, France \\ ${ }^{2}$ Laboratory of Fluid Dynamics and Seismics, Moscow Institute of Physics and Technology (State University), \\ Dolgoprudny 141700, Russia
}

Correspondence should be addressed to Mikhail Panfilov; mikhail.panfilov@univ-lorraine.fr

Received 3 October 2015; Revised 4 January 2016; Accepted 5 January 2016

Academic Editor: Mohammad Al-Nimr

Copyright (C) 2016 M. Panfilov and A. Koldoba. This is an open access article distributed under the Creative Commons Attribution License, which permits unrestricted use, distribution, and reproduction in any medium, provided the original work is properly cited.

To describe phase equilibrium of mixtures, we develop a nonclassical approach based on using different equations of state for gas and liquid. We show that not all the types of EOS are admissible but only those which verify some specific conditions of consistency. We developed the mathematical theory of this new approach for pure cores and for mixtures, in presence and absence of capillary forces, which leads to explicit analytical relationships for phase concentrations of chemical components. Several examples of comparison with experimental data for binary and ternary mixtures illustrate the feasibility of the suggested approach.

\section{Introduction}

The objective of the present paper is to develop simplified mathematical models of phase equilibria which would significantly reduce computation time, while retaining an acceptable level of precision. This problem is highly pertinent for multicomponent two-phase or three-phase fluids. The results of such simulations provide information about the chemical composition of each phase at any pressure and temperature and the fractional volume of each phase.

Modern thermodynamic models are essentially based on cubic equations of state (EOS) such as those developed by Van-der-Waals, Peng-Robinson, or Redlich-Kwong [1-4]. They ensure high quality in descriptions of gas-liquid coexistence, which determines the success of the modern thermodynamic theory. At the same time, when such models are used to simulate fluid flow in geological oil, gas, or water reservoirs, more than the half of the computation time is spent on the thermodynamic block. The iterative numerical procedure suggested in $[5,6]$ in a form called "the flash calculation" must be done at each time step and at each space point which is highly time-consuming.
Consequently a lot of papers have been published with the objective of reducing the time of flash calculation by reducing the number of primary variables. Michelsen in [7] reduced the number of parameters to three and accepted zero values for all binary interaction parameters (BIPs). By only taking into account the BIPs between a single component and the system of all other components, the number of parameters was reduced to five in [8]. More recently, in [9] the authors suggested the split of the BIPs into two parameters using a simple quadratic expression.

Unfortunately the simplest approaches based on empirical Henry's or Raoulth's laws only permit the calculation of the composition of one phase and are not adapted for use with multiphase multicomponent systems.

Another way to reduce the computational time for the general coupled hydrodynamic-thermodynamic problem consists of splitting the thermodynamics and hydrodynamics. An example of such splitting is published in $[10,11]$, where the authors developed the so-called HT-splitting, an asymptotic procedure providing nonclassic thermodynamic equations completely independent of hydrodynamic equations. This 
approach has lead to a new kind of thermodynamic model made up of traditional algebraic relationships and new differential thermodynamic equations.

Completely different technique of splitting the thermodynamics and hydrodynamics was developed in [12] for mono-dimensional two-phase $n$-component flow. By a specific replacement of variables, the $n \times n$ conservation laws are transformed into a reduced $(n-1) \times(n-1)$ closed auxiliary system containing just thermodynamic variables and one lifting hydrodynamic equation containing the phase saturation.

A cubic EOS has to be excessively complicated in order to be capable of capturing not only the single-phase behaviour of gas and liquid but also the transition two-phase zone. However, just within this zone the cubic EOS is not used: its complicated nonmonotonic behaviour is simply replaced by an isobaric straight line (Maxwell's line) which corresponds to the true gas-liquid equilibrium. Then our logic was the following: it is not necessary to require that an EOS would describe the transition zone; then it is possible to describe liquid and gas by two different EOS, which may represent very simple equations. This becomes the significant source of simplifying equilibrium equations.

The system of equilibrium equations and two different EOS for gas and liquid will be called the dissymmetric equilibrium model.

The approach based on dissymmetric EOS is not new and is frequently applied in chemistry for various purposes. A lot of other papers exist when the dissymmetric models are applied to calculate some thermodynamic parameters of mixtures. Their application to calculate phase equilibria is less known. This was done first probably by Kelvin, who used the model of the ideal gas and an incompressible liquid to describe the equilibrium controlled by capillary forces for a monocomponent fluid. The dissymmetric EOS for two-component fluids was applied in [13] to calculate the two-phase envelop. In [14] the generalization of the Kelvin equation was obtained for multicompositional mixtures by applying the real-gas EOS and an incompressible liquid and by introducing the new concept of a "mixed volume." The resulting equation is valid only near the dew point.

Another kind of dissymmetric models, known as the phigamma approach, has been used for decades in chemical engineering thermodynamics [15]. In this approach vapor is described by an EOS, while liquid is described by the activity coefficient. The model used for the activity coefficient represents some semiempirical approximations but never an EOS.

The main difference of the present paper from all other papers in which a kind of dissymmetric approaches was applied consists of the following:

(i) Within the present paper we use two different EOS for gas and liquid to calculate phase equilibria.

(ii) We have shown that not any couple of EOS is consistent with the two-phase equilibrium.

(iii) We have determined the general theoretical constraints to the choice of two EOS consistent with the gas-liquid equilibrium.
In all the papers which used the dissymmetric approach the selection of the individual EOS is arbitrary and not constrained. However, once we tried to develop dissymmetric EOS models and to extend them to multicomponent mixtures, we found that several EOS lead to nonexistence of two-phase states. In particular, it is possible to show that the incompressible liquid is unable to capture two-phase states whatever the EOS for gas is.

The capacity of an EOS to capture two-phase states is called consistency conditions. Thus, the selection of the individual dissymmetric EOS for gas and liquid is not arbitrary but must satisfy the consistency conditions which represent mathematically the conditions of solution existence for the equilibrium equations.

The development of the consistency conditions and their use in order to determine the adjustable parameters of the dissymmetric EOS is the second key element of the paper.

The developed approach gives a form of equilibrium equations which can be solved analytically with respect to phase concentrations even for multicomponent nonideal systems, which significantly reduces the computational time.

\section{Principle Idea: One-Component Fluid}

The best way of presenting the principles of our approach is to analyze a simple fluid consisting of one chemical component.

2.1. Main Equilibrium Relationship. The gas-liquid equilibrium may be described using the system of one equilibrium equation, (1a), and two equations of state, (1b) and (1c), which have the following form:

$$
\begin{aligned}
& \int_{P_{r g}}^{P_{\mathrm{eq}}} v_{g}(P, T) d P+\mu_{g}\left(P_{r g}, T\right) \\
& \quad=\int_{P_{r l}}^{P_{\mathrm{eq}}} v_{l}(P, T) d P+\mu_{l}\left(P_{r l}, T\right), \\
& v_{g}=v_{g}(P, T), \\
& v_{l}=v_{l}(P, T),
\end{aligned}
$$

where $P$ is the pressure, $T$ is the temperature, $v$ is the molar volume, $\mu$ is the chemical potential, $P_{r g}$ and $P_{r l}$ are two arbitrary reference values of pressure, $P_{\mathrm{eq}}$ is the equilibrium pressure at given $T$, and indexes $g$ and $l$ correspond to gas and liquid, respectively.

The definition of the chemical potential, the fluid volume, and the molar volume through the Gibbs energy $G$ is

$$
\begin{aligned}
\mu & =\frac{\partial G}{\partial N}, \\
V & =\frac{\partial G}{\partial P}, \\
v & =\frac{\partial V}{\partial N},
\end{aligned}
$$

where $N$ is the number of moles of fluid. 
Proof of (1a). The most general conditions of equilibrium for simple fluid (without capillarity) are

$$
\begin{gathered}
P_{g}=P_{l} \equiv P_{\mathrm{eq}}, \\
T_{g}=T_{l} \equiv T, \\
\mu_{g}\left(P_{\mathrm{eq}}, T\right)=\mu_{l}\left(P_{\mathrm{eq}}, T\right) .
\end{gathered}
$$

Using definition (2) and the equivalence, $(\partial / \partial P)(\partial G /$ $\partial N)=(\partial / \partial N)(\partial G / \partial P)$, we obtain

$$
\frac{\partial \mu}{\partial P}=\frac{\partial V}{\partial N}=v
$$

which yields the well known explicit relationship for the chemical potential:

$$
\mu(P, T)=\int_{P_{r}}^{P} v(\bar{P}, T) d \bar{P}+\mu\left(P_{r}, T\right) .
$$

Substituting (5) in the equilibrium conditions (3) we obtain (1a).

In system ((1a)-(1c)) the chemical potentials $\mu_{g}\left(P_{r g}, T\right)$ and $\mu_{l}\left(P_{r l}, T\right)$ are known at reference pressures $P_{r g}$ and $P_{r l}$.

Then, for any $T$, (1a) represents the nonlinear system of one equation with respect to $P_{\mathrm{eq}}$ which is the pressure of gasliquid coexistence.

The classic approach consists of using the same EOS for all fluids, whereas our approach consists of using different EOS for gas and for liquid.

2.2. Pseudoliquid and Pseudogas. Within the framework of the suggested approach, it is necessary to introduce the following definitions.

The pseudoliquid and the pseudogas are the hypothetical fluids which are authorized to exist at any $P$ and $T$, with each of them being defined only by a formal EOS, (1b) or (1c).

This means that both pseudophases can coexist at any $P$ and $T$, but this kind of coexistence is not proved by any equilibrium conditions.

Figure 1 presents two curves, ADE and ABM, which are the graphs of the EOS for pseudogas and pseudoliquid, respectively.

The true coexisting gas and liquid are the fluids defined by the system of two EOS, (1b) and (1c), and the equilibrium equation, (1a), in such a way that this system of three equations can only have one solution for the coexistence pressure $P_{\text {eq }}$ and molar volumes $v_{g \text {,eq }}$ and $v_{l, \text { eq }}$ (at a fixed temperature $T$ ) which satisfy the strong inequality:

$$
v_{g, \mathrm{eq}}>v_{l, \mathrm{eq}}
$$

In Figure 1, this corresponds to the horizontal line of Maxwell $B D$, which is characterized by three parameters: $P_{\text {eq }}$, $v_{l, \mathrm{eq}}$, and $v_{g, \mathrm{eq}}$.

The true gas and liquid are the fluids which are determined only by the EOS and exist outside the two-phase coexistence domain; that is, the true gas corresponds to $v_{g} \geq v_{g \text {, eq }}$, while the true liquid corresponds to $v_{l} \leq v_{l, \text { eq }}$.

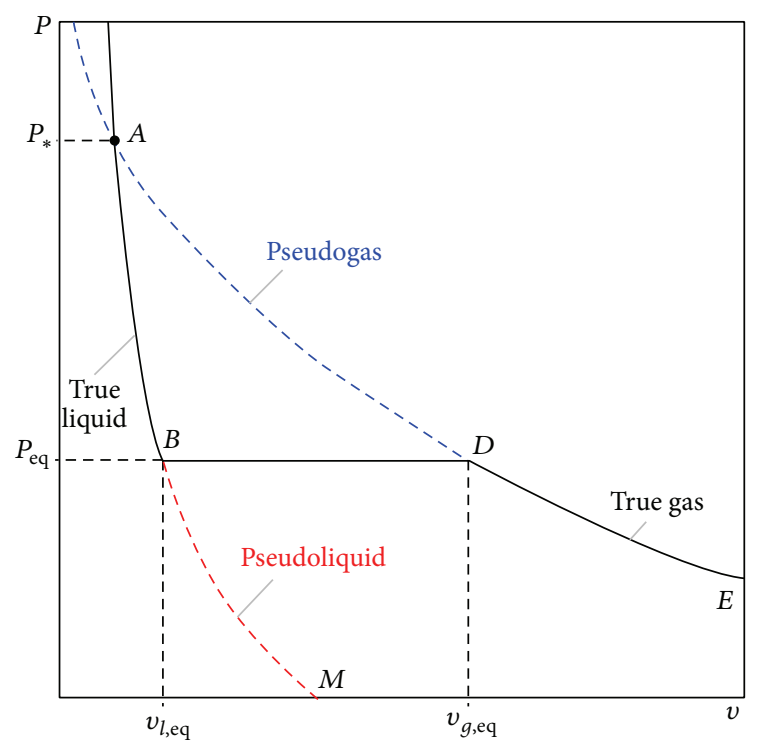

FIGURE 1: Illustration of the concept of the true and pseudophases in the diagram $P-v$.

In Figure 1, this corresponds to the segment $\mathrm{DE}$ for the true gas and BA for the true liquid.

Both the true phases and pseudophases are described by the same EOS. This means that a pseudoliquid may be considered as the continuous prolongation of the true liquid into the domain of molar volumes greater than $v_{l, \text { eq }}$, while the pseudogas is the prolongation of the true gas into the domain of volumes lower than $v_{g \text {,eq }}$.

2.3. Pseudocritical Point. Two curves $v(P)$ described by two independent EOS can cross one another at a point $(A)$, which corresponds to pressure $P_{*}$. At this point the pressure, temperature, and volume of gas and liquid are trivially identical, since the difference between gas and liquid disappears. Consequently, the chemical potentials in $A$ are identical. This can be proved more strictly in three different ways.

Thermodynamic Method. At point $A$ we have two substances, for which all the parameters of state are identical: pressure, volume, temperature, and composition. Then each function of state is also identical for them. The chemical potential is the internal energy reported to one mole, and the internal energy is the function of state. Then the chemical potentials of two substances are identical.

Molecular Physics Method. At point $A$ we have two substances, which have identical molecules (the same composition), identical distances between the molecules (identical pressure and volume), and identical fluctuation energy (identical temperature). Then the internal energy of these two substances is identical. Then their chemical potentials are also identical.

Mathematical Method. Let us use the property of homogeneity of the Gibbs energy. Indeed, the Gibbs free energy depends on fluid pressure $P$, temperature $T$, volume $V$, and 
the number of moles $N: G=G(P, T, V, N)$. Being an additive function of the number of particles, the energy satisfies the following homogeneity condition: $G(P, T, \lambda V, \lambda N)=$ $\lambda G(P, T, V, N)$, for any $\lambda$. Selecting $\lambda=1 / N$, we obtain

$$
G\left(P, T, \frac{1}{N} V\right)=\frac{1}{N} G(P, T, V, N) .
$$

The Euler theorem for homogeneous functions says that

$$
\begin{gathered}
\frac{1}{N} G(P, T, V, N)=\frac{\partial G(P, T, V, N)}{\partial N} \equiv \mu, \\
\frac{1}{N} V(N)=\frac{\partial V(N)}{\partial N} \equiv v .
\end{gathered}
$$

It follows from (7) and (8) that $\mu=\mu(P, T, v)$.

Thus, for fixed values of $P, T, v$, the chemical potential of a fluid, $\mu$, is a constant value, independently of its phase state. Then we have the following at point $P_{*}: \mu_{g}=\mu_{l}$.

Respectively, point $P_{*}$ will be called pseudocritical. Pressure $P_{*}(T)$ is the pseudocritical pressure which is defined as the solution of the following equation:

$$
v_{l}\left(P_{*}, T\right)=v_{g}\left(P_{*}, T\right) .
$$

In general case, two monotonically decreasing curves can cross each other at several points: $P_{*}^{\mathrm{I}}, P_{*}^{\mathrm{II}}, \ldots$. All of them will be called pseudocritical.

If we select for the reference pressures, $P_{r g}=P_{r l}=P_{*}$, then the chemical potentials $\mu_{g}\left(P_{*}, T\right)$ and $\mu_{l}\left(P_{*}, T\right)$ must be identical. Consequently, the equilibrium equation ((1a)-(1c)) becomes

$$
\int_{P_{*}}^{P_{\text {eq }}}\left[v_{g}(P, T)-v_{l}(P, T)\right] d P=0
$$

which is the algebraic equation with respect to the equilibrium pressure $P_{\text {eq }}$.

The geometrical meaning of the left-hand side is the surface between two curves $v_{g}(P)$ and $v_{l}(P)$ (at a fixed $T$ ), calculated between the pseudocritical pressure $P_{*}$ and the pressure of gas-liquid coexistence $P_{\text {eq }}$.

2.4. Application to the Case of a Uniform EOS for Both Phases. In the traditional case, the EOS is identical both for liquid and for gas. Then any point corresponds to (9) and is pseudocritical. It is easy to show that in this case equilibrium equation (10) is independent of the selection of $P_{*}$.

Let $P_{*}$ be an arbitrary reference pressure located far from the two-phase zone. Let us transform the integration $\int v(P) d P$ in (10) to $\int P(v) d v$. The integration by parts of (10) leads to

$$
\begin{aligned}
& \left.v P\right|_{v_{*} P_{*}} ^{v_{\text {geq }} P_{\text {eq }}}-\int_{v_{*}}^{v_{g, \text { eq }}} P(v) d v \\
& =\left.v P\right|_{v_{*} P_{*}} ^{v_{\text {leq }} P_{\text {eq }}}-\int_{v_{*}}^{v_{\text {leq }}} P(v, T) d v
\end{aligned}
$$

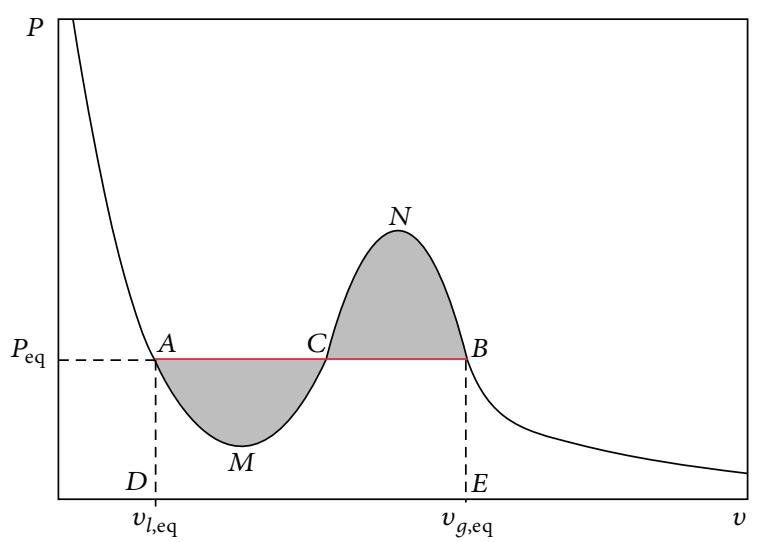

FIGURE 2: Graphical technique of determining the equilibrium line in the case of using the uniform (cubic) EOS for gas and liquid.

or

$$
\begin{aligned}
\left(v_{g, \text { eq }}-v_{l, \text { eq }}\right) P\left(v_{g, \text { eq }}\right) & =\int_{v_{\text {leq }}}^{v_{g, \text { eq }}} P(v) d v, \\
P\left(v_{g, \text { eq }}\right) & =P\left(v_{l, \text { eq }}\right)
\end{aligned}
$$

which is the system of two nonlinear equations for two equilibrium molar volumes: $v_{g, \text { eq }}$ and $v_{l \text {,eq }}$.

For a cubic EOS, as the Van-der-Waals equation, $P=$ $R T /(v-b)-a / v^{2}$, with two empirical coefficients, $a$ and $b$, a curve $P(v)$ below the critical point has the form shown in Figure 2. Then the existence of the nontrivial solution of the first equation in (12) may be interpreted graphically as shown in Figure 2. The left-hand side of this equation is the rectangular area $A B D E$. The right-hand side obviously corresponds to the same area if the grey areas $A M C$ and $\mathrm{CNB}$ eliminate one another, that is, are identical, which determines the unique location of the Maxwell line $A B$ and the equilibrium pressure $P_{\mathrm{eq}}$.

This graphical technique is known as Maxwell's equal area rule.

The Maxwell rule determines the mathematical necessary condition on the behaviour of the curve $P(v)$ which ensures the existence of two-phase states: it must be nonmonotonic with $M$ minimums and $M$ maximums, where $M \geq 1$. Only in this case does system (12) have a nontrivial solution. This property of the curve $P(v)$ represents the condition of consistency between the EOS and the equilibrium equation. Obviously, this property is verified for a cubic EOS with respect to volume.

2.5. Consistency Conditions. For a dissymmetric model, the technique of application of the equilibrium equation (10) and the consistency condition are different and are sufficiently based on using the pseudocritical pressure $P_{*}$.

The existence of two-phase state imposes some constraints on the form of two individual EOS which should ensure the solvability of (10) with respect to $P_{\text {eq }}$. We will call these constraints the solvability conditions of the equilibrium 


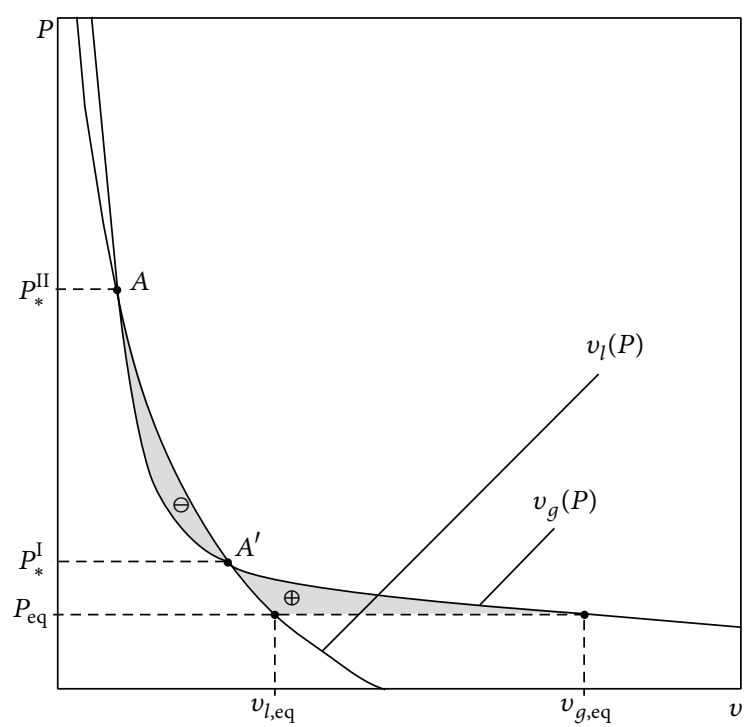

(a)

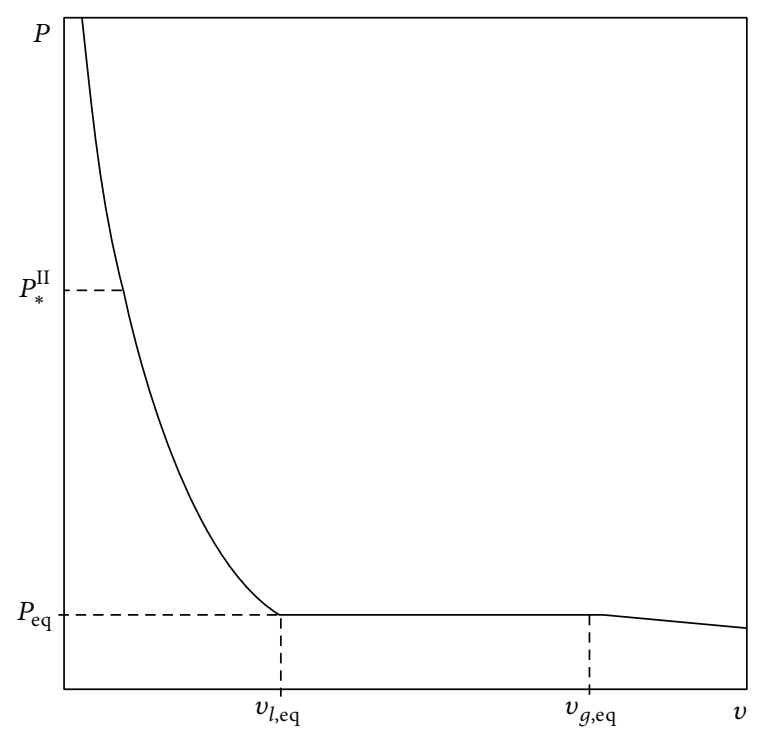

(b)

FIGURE 3: (a) Graphical interpretation of the consistency conditions: the gray area corresponds to the integral in (10). (b) The resulting phase diagram.

equation or consistency conditions between the equilibrium equation and EOS.

The necessary conditions of solvability may be formulated in a geometrical way, taking into account the fact that the integral in (10) represents the surface between two curves $v_{g}(P)$ and $v_{l}(P)$ (at a fixed $T$ ), as shown in Figure 3(a).
In order to be zero, this surface has to have at least one positive part and one negative part and the areas of these two parts must be identical. Thus, we obtain the necessary and sufficient criteria of solvability or the consistency conditions:

At fixed $T$,

$$
\begin{gathered}
v_{g}(P)-v_{l}(P)=0 \quad \text { has two different positive solutions: } P_{*}^{\mathrm{I}} \text { and } P_{*}^{\mathrm{II}}\left(P_{*}^{\mathrm{II}}>P_{*}^{\mathrm{I}}>0\right) ; \\
v_{g}>v_{l}, \quad \forall P \in\left(P_{*}^{\mathrm{I}}, P_{*}^{\mathrm{II}}\right) .
\end{gathered}
$$

The first condition means that the curves $v_{g}(P)$ and $v_{l}(P)$ cross each other twice (points $A$ and $A^{\prime}$ in Figure 3 ). This is necessary to obtain two segments with different signs of the area between these two curves.

The second condition (which is valid only for nontrue gas and liquids) determines the correct placing of these two curves with respect to one another in order to ensure inequality of (6) for true gas and liquid.

Then the equilibrium equation (10) takes the more exact form:

$$
\int_{P_{*}^{\mathrm{II}}}^{P_{\mathrm{eq}}}\left[v_{g}(P, T)-v_{l}(P, T)\right] d P=0 .
$$

If the conditions ((13a) and (13b)) are satisfied, then the equilibrium pressure $P_{\mathrm{eq}}$ is determined from (14) in such a way that the negative and positive areas of the surface between the curves $v_{g}(P)$ and $v_{l}(P)$ are identical between points $P_{*}^{\mathrm{II}}$ and $P_{\text {eq }}$.
In general case, two curves $v_{g}(P)$ and $v_{l}(P)$ can cross each other at a number of points larger than two. But to ensure solvability, it is sufficient to have only two points.

From this graphical technique it follows that the twophase equilibrium is impossible for an incompressible liquid. Indeed, any monotonic function $v_{g}(P)$ cannot cross twice the vertical line $v_{l}=$ const.

From these principles, we deduced the following graphical technique which may be easily used to detect the consistency (or inconsistency) of the selected system of EOS and to determine the equilibrium pressure:

(i) Two EOS whose plots $P$ versus $v$ satisfy the following conditions need to be selected:

(a) both functions $P(v)$ for gas and liquid should be monotonic at each point $v$;

(b) they cross each other at two different pressures $P_{*}^{\mathrm{I}}$ and $P_{*}^{\mathrm{II}}$ (Figure 3 ); 
(c) the curve $P(v)$ for pseudogas is situated on the left from that of pseudoliquid between $P_{*}^{\mathrm{I}}$ and $P_{*}^{\mathrm{II}}$, according to condition (13b).

(ii) The equilibrium pressure $P_{\text {eq }}$ is determined graphically in such a way that the surface of the area between two curves between points $P_{*}^{\mathrm{I}}$ and $P_{\text {eq }}$ (Figure 3) is identical to the area of the surface between points $P_{*}^{\mathrm{I}}$ and $P_{*}^{\mathrm{II}}$.

In the classical case considered in the previous section the equilibrium equations (12) are solved with respect to two different volumes $v_{l, \text { eq }}$ and $v_{g \text {,eq }}$. In contrast to it, the solution of (10) is searched directly with respect to the unique value of pressure $P_{\text {eq }}$.

The graphical areas which eliminate one another are located along axis $P$ (Figure 3(a)) and not axis $v$, which represents reversed Maxwell's rule; that is, the analogue of the Maxwell rule of equal areas turned by $90^{\circ}$ counterclockwise.

2.6. Consistent Exponential EOS for Liquid and Ideal EOS for Gas. Another model which satisfies the consistency conditions is that of the ideal EOS for gas and the exponential EOS for liquid, which represents a theoretical equation based on the assumption that the compressibility coefficient is independent of pressure:

$$
\begin{aligned}
v_{g} & =\frac{R T}{P}, \\
v_{l} & =v_{l 0} e^{-\beta\left(P-P_{0}\right)},
\end{aligned}
$$

where $R$ is the universal gas constant value, $P_{0}$ is an arbitrary constant pressure value (different from $P_{r}$ in (1a)-(1c) and (5)), while $v_{l 0}(T)$ and $\beta(T)$ are two empirical parameters of the model which determine the behaviour of the phase diagram. Parameter $\beta$ has the meaning of the isothermal compressibility coefficient. Indeed, the exponential equation results immediately from the definition of the compressibility coefficient: $\beta \equiv-(1 / v)(d v / d P)$, if we assume that $\beta=\beta(T)$, which is valid far from the critical point.

Let us prove that the selected model (15) is self-consistent, if the parameters verify the following conditions:

$$
0<a<e^{-1}=0.37, \quad a \equiv \beta P_{\oplus}, P_{\oplus} \equiv \frac{R T}{v_{l 0}} e^{-\beta P_{0}} .
$$

Indeed, (13a) takes the following dimensionless form through the dimensionless pressure $p \equiv P / P_{\oplus}$ :

$$
p=e^{a p} \text {. }
$$

The plot of the left-hand and right-hand sides is shown in Figure 4 .

Two solutions $p_{*}^{\mathrm{I}}$ and $p_{*}^{\mathrm{II}}$ transform to a single value if the exponential function $y_{1}=e^{a p}$ touches the straight line $y_{2}=p$, which means the identical derivatives of $y_{1}$ and $y_{2}$ : $a e^{a p}=1$. This equation and (17) determine two critical values of $p$ and $a$ :

$$
\begin{aligned}
& a_{\mathrm{cr}}=e^{-1} \\
& p_{\mathrm{cr}}=e
\end{aligned}
$$

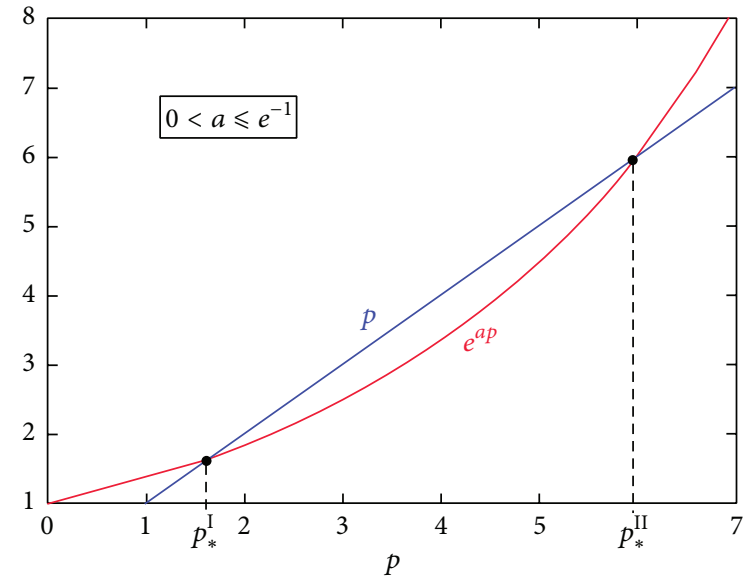

FIGURE 4: Solvability of (17).

which corresponds to the upper estimation in (16). For larger values of $a$, two curves do not cross each other, which means that (17) has no solutions.

For smaller values of $a$, the curve $y_{1}=e^{a p}$ crosses the line $y_{2}=p$ twice at two points $p_{*}^{\mathrm{I}}$ and $p_{*}^{\mathrm{II}}$. When $a \rightarrow 0$, the first pressure $p_{*}^{\mathrm{I}}$ tends to 1 and the second pressure $p_{*}^{\mathrm{II}}$ tends to infinity. This yields the lower estimation in (16), which corresponds to the incompressible liquid. Thus, for these values of $a$, condition (13a) is satisfied. It is easy to show that $(13 b)$ is also satisfied.

Once the pseudocritical pressure $p_{*}^{\mathrm{II}}$ is determined from (17), the equilibrium pressure, $p_{\mathrm{eq}}$, is found as the solution to the following equation resulting from (14):

$$
a \ln \frac{p_{\mathrm{eq}}}{p_{*}^{\mathrm{II}}}=e^{-a p_{*}^{\mathrm{II}}}-e^{-a p_{\mathrm{eq}}}
$$

The value of $p_{\mathrm{eq}}$ is the nontrivial solution of this equation, different from $p_{*}^{\mathrm{II}}$.

So, for given $a$, one can get $p_{*}^{\mathrm{II}}$ and $p_{\text {eq }}$. Since we know the gas and liquid EOS we can get $v_{l, \text { eq }}$ and $v_{g \text {,eq }}$ and, finally, the envelope of the phase diagram (this being the line joining all the dew points and all the bubble points).

\section{Determination of the Coefficients}

3.1. General Technique of Adjusting Dissymmetric Models. As shown in Section 2.6, for ideal gas and exponential EOS for liquid, if we know the values of $\beta$ and $v_{l 0}$ (i.e., the parameter a), then it is possible to determine the equilibrium pressure and volumes. In the similar way it is possible to formulate the symmetrical problem: to determine parameters $\beta(T)$ and $v_{l 0}(T)$ by using the experimental information on the equilibrium pressure and volumes of pure components. The obtained adjusted dissymmetric models will be used further to describe multicomponent mixtures without any additional fitting (Section 4). 
We can extend this approach by using the virial EOS for gas in the form

$$
v_{g}=\frac{R T}{P}+v_{g 0},
$$

where the new parameter $v_{g 0}$ should be adjusted by using the data on the equilibrium gas volume, $v_{g \text {,eq }}$.

The virial EOS gives more accurate results for equilibrium gas volume than the ideal gas EOS.

Let us assume that the equilibrium pressure $P_{\text {eq }}$, the equilibrium molar liquid, and gas volumes $v_{l, \text { eq }}$ and $v_{g \text {,eq }}$ are given as experimental data. For pure components, they can be found in the literature or calculated by Peng-Robinson EOS.

The objective is to determine the values of $\beta, v_{l 0}, v_{g 0}$, and $P_{*}^{\mathrm{II}}$. Parameter $P_{0}$ is selected in a free way (in the examples below it was chosen as $P_{0} \approx 2 P_{c}$ ). Then the system of (17), (19), and (20) and the second equation in (15) are sufficient to determine $\beta, v_{l 0}, v_{g 0}$, and $P_{*}^{\mathrm{II}}$ through $P_{\mathrm{eq}}, v_{l, \mathrm{eq}}$, and $v_{g, \mathrm{eq}}$. This system is

$$
\begin{aligned}
& v_{g 0}\left(P_{\mathrm{eq}}-P_{*}^{\mathrm{II}}\right)+R T \ln \frac{P_{\mathrm{eq}}}{P_{*}^{\mathrm{II}}} \\
& =-\frac{v_{l 0}}{\beta}\left(e^{\beta\left(P_{0}-P_{\mathrm{eq}}\right)}-e^{\beta\left(P_{0}-P_{*}^{\mathrm{II}}\right)}\right), \\
& v_{g 0}+\frac{R T}{P_{*}^{\mathrm{II}}}=e^{\beta\left(P_{0}-P_{*}^{\mathrm{II}}\right)}, \\
& v_{g, \text { eq }}=v_{g 0}+\frac{R T}{P_{\mathrm{eq}}}, \\
& v_{l, \text { eq }}=v_{l 0} e^{\beta\left(P_{0}-P_{\mathrm{eq}}\right)} .
\end{aligned}
$$

Introducing new dimensionless variables,

$$
\begin{aligned}
\mathscr{Q} & \equiv \beta P_{\mathrm{eq}}, \\
\mathscr{P} & \equiv \frac{P_{*}^{\mathrm{II}}}{P_{\mathrm{eq}}}, \\
\xi & \equiv \frac{R T}{v_{l, \mathrm{eq}} P_{\mathrm{eq}}}, \\
\eta & \equiv \frac{v_{g, \mathrm{eq}}}{v_{l, \mathrm{eq}}},
\end{aligned}
$$

we can transform system (21) to one nonlinear equation which determines the function $\mathscr{P}(\xi, \eta)$, where $\xi$ and $\eta$ are the given parameters,

$$
\begin{aligned}
& \frac{1}{1-\mathscr{P}} \ln \left(\eta-\xi+\frac{\xi}{\mathscr{P}}\right)[(\eta-\xi)(1-\mathscr{P})-\xi \ln \mathscr{P}] \\
& =\eta-\xi+\frac{\xi}{\mathscr{P}}-1,
\end{aligned}
$$

and an explicit relationship for function $\mathbb{Q}(\xi, \eta)$ :

$$
\mathscr{Q}=\frac{1}{1-\mathscr{P}} \ln \left(\eta-\xi+\frac{\xi}{\mathscr{P}}\right) \text {. }
$$

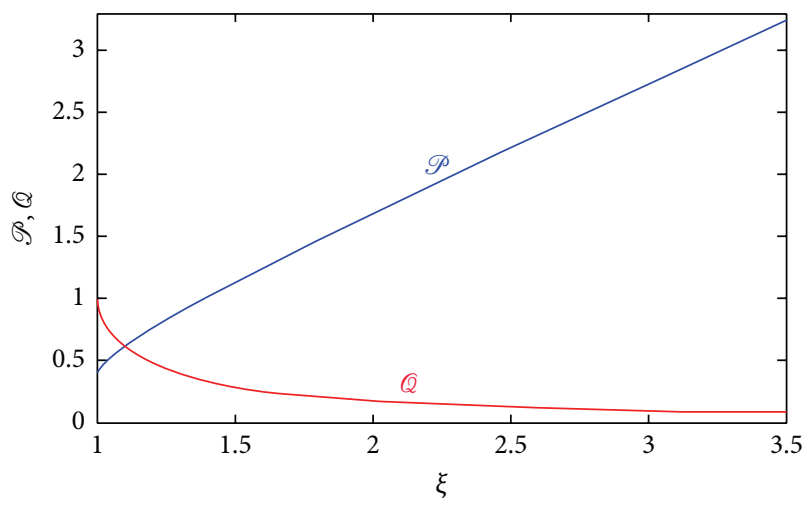

Figure 5: Functions $\mathscr{P}(\xi)$ and $\mathscr{Q}(\xi)$ defined by (25).

If the gas phase is ideal, then $\eta=\xi$; then we obtain for $\mathscr{P}(\xi)$ and $\mathbb{Q}(\xi)$ instead of $(23)$

$$
\begin{aligned}
& -\frac{\mathscr{P}}{\mathscr{P}-1} \ln \left(\frac{\mathscr{P}}{\xi}\right) \ln \mathscr{P}=1-\frac{\mathscr{P}}{\xi}, \\
& \mathbb{Q}=\frac{1}{1-\mathscr{P}} \ln \left(\frac{\xi}{\mathscr{P}}\right) .
\end{aligned}
$$

Proof. (1) Eliminating $v_{l 0}$ and $v_{g 0}$ from the last two equations (21), we obtain from the first two equations

$$
\begin{aligned}
(\eta-\xi) \mathscr{Q}(1-\mathscr{P})-\xi \mathscr{Q} \ln \mathscr{P} & =e^{\mathscr{Q}(1-\mathscr{P})}-1, \\
\eta-\xi+\frac{\xi}{\mathscr{P}} & =e^{\mathscr{Q}(1-\mathscr{P})} .
\end{aligned}
$$

(2) Let us eliminate $Q$ from the second relationship, which gives (24). Substituting it into the first equation, we deduce (23).

Let us note that the functions $\mathscr{P}(\xi, \eta)$ and $\mathscr{Q}(\xi, \eta)$ are universal and do not depend on individual properties of chemical components.

The solution of (25) obtained numerically is shown in Figure 5.

To select the initial approximation for iterative solution of (25), it is possible to use the coarse approximation obtained by fitting the curve shown in Figure 5: $\mathscr{P} \approx \xi^{0.98}$; then $\mathscr{Q} \approx$ $\left(0.02 /\left(1-\xi^{0.98}\right)\right) \ln \xi$.

Note that the boundary values of these functions which correspond to the formal critical point are $\mathbb{Q}_{\mathrm{cr}}=1, \mathscr{P}_{\mathrm{cr}}=1$, and $\xi_{\mathrm{cr}}=1$.

Using $\mathscr{P}$ and $\mathscr{Q}$, we can determine parameters $\beta, P_{*}^{\mathrm{II}}, v_{l 0}$, and $v_{g 0}$ :

$$
\begin{aligned}
\beta & =\frac{\mathscr{Q}}{P_{\mathrm{eq}}}, \\
P_{*}^{\mathrm{II}} & =\mathscr{P} P_{\mathrm{eq}}, \\
v_{l 0} & =v_{l, \mathrm{eq}} e^{\beta\left(P_{\mathrm{eq}}-P_{0}\right)}, \\
v_{g 0} & =v_{g, \mathrm{eq}}-\frac{R T}{P_{\mathrm{eq}}} .
\end{aligned}
$$




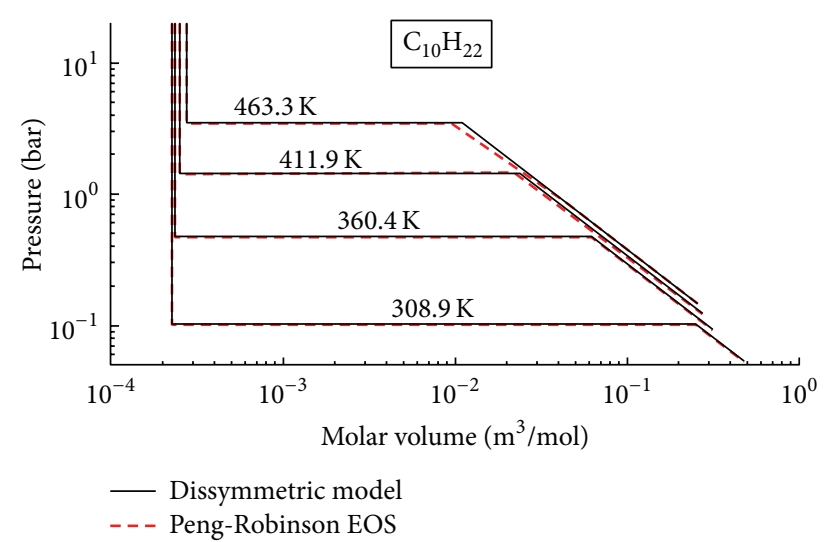

FIgURE 6: $P-v$ curves for Decane by dissymmetric model (the black curves) and through Peng-Robinson EOS (the red dashed curves).

3.2. Example of Fitting. As the example of application of this technique, we present the results obtained for Decane for four temperatures: $T=\{308.9,360.4,411.9,463.3\} \mathrm{K}$. The critical pressure and temperature for it are $2.11 \mathrm{MPa}$ and $617.8 \mathrm{~K}$. The exact data on the equilibrium pressure and liquid volume have been calculated by using the Peng-Robinson equation of state.

The values of parameter $\xi$ are calculated from its definition; parameter $\mathscr{P}$ is calculated as the solution to (25). The reference pressure was selected as $P_{0}=2 P_{c}$. The obtained values of the coefficient of compressibility $\beta$ are $\left(\mathrm{MPa}^{-1}\right)$ as follows:

$$
\left\{0.802 \cdot 10^{-8} ; 0.851 \cdot 10^{-8} ; 0.919 \cdot 10^{-8} ; 1.021 \cdot 10^{-8}\right\}
$$

and the reference molar liquid volume $v_{l 0}$, calculated from (27), is $\left(\mathrm{m}^{3} / \mathrm{mol}\right)$ :

$$
\{0.0002187 ; 0.0002303 ; 0.0002459 ; 0.0002679\} \text {. }
$$

The results are presented in Figure 6.

In Figure 7, we present the diagrams for several pure components calculated by the dissymmetric model and PengRobinson EOS, for $T=300 \mathrm{~K}$.

Remarks. (1) The developed technique of determination of the parameters gives unsatisfactory results for temperatures close to the critical point. For the moment, the dissymmetric models cannot pretend to be a good tool to describe the critical point. Indeed, the true critical point is the inflexion point and the point of zero derivative for the true curve $P(v)$. Such a complex behaviour is impossible for the point of intersection of two monotonic curves which correspond to pseudogas and pseudoliquid.

(2) In the present paper, we use the ideal EOS for gas just to simplify the principle ideas of the suggested approach. Obviously, the ideal EOS can lead to some errors in calculating the equilibrium gas volume, especially at high equilibrium pressures. The virial EOS (20) gives more adequate results.

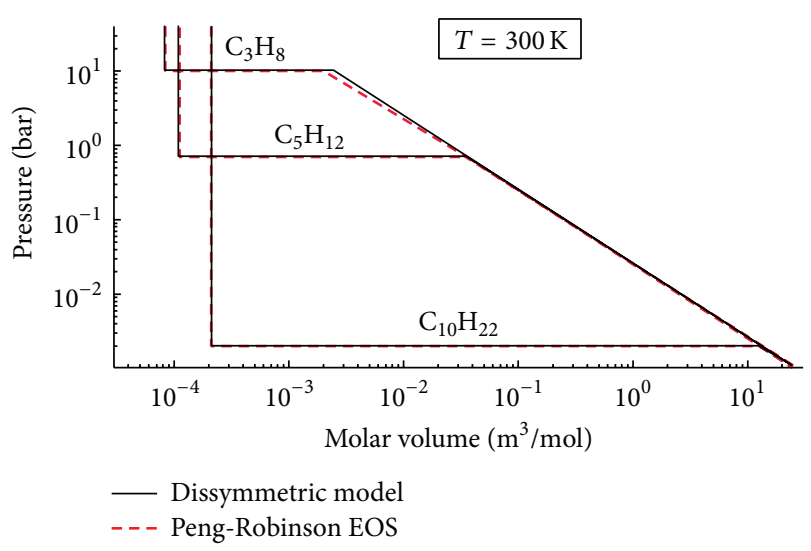

FIgURE 7: $P-v$ curves for three pure components by dissymmetric model (the black curves) and through Peng-Robinson EOS (the red dashed curves).

\section{Equilibrium of Multicomponent Phases}

Let us suppose that the fluid consists of $n$ chemical components which are present in gas and liquid. The gas-liquid equilibrium would now determine the composition of each phase and not just the equilibrium pressure and volumes.

The superscript $k$ will be the number of chemical components; the superscript "pure" refers to the pure component considered at the same pressure and temperature as the mixture; the subscript "eq" refers to the gas-liquid equilibrium. Other notations are as follows:

(i) $c_{\alpha}^{k}$ is the mole fraction of component $k$ in phase $\alpha$, which will be also called "the phase concentration."

(ii) $C^{k}$ is the mole fraction of component $k$ in the entire fluid, which will be also called "the total concentration."

(iii) $\mu_{\alpha}^{k}$ is the chemical potential of component $k$ in phase $\alpha$.

(iv) $\mu^{k \text {,ideal }}$ is the chemical potential of component $k$ in the mixture if the mixture behaves as an ideal gas.

(v) $\mu^{k \text {, pure }}$ is the chemical potential of the pure component $k$ if it behaves as an ideal gas.

(vi) $N_{\alpha}^{k}$ is the number of moles of component $k$ in phase $\alpha$.

(vii) $V_{\alpha}$ is the volume of phase $\alpha$.

(viii) $v_{\alpha}^{k} \equiv \partial V_{\alpha} / \partial N_{\alpha}^{k}$ is the partial molar volume of component $k$ in phase $\alpha$.

(ix) $v_{\alpha} \equiv \partial V_{\alpha} / \partial N_{\alpha}$ is the total molar volume of phase $\alpha$.

(x) $P_{*}^{\mathrm{I}, k}$ and $P_{*}^{\mathrm{II}, k}$ are two pseudocritical pressures of pure component $k$.

(xi) $P_{\text {eq }}^{k}$ is the pressure of gas-liquid coexistence for pure component $k$.

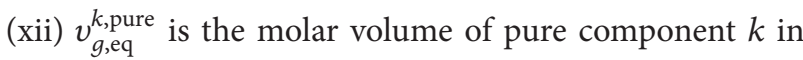
equilibrium gas state. 
(xiii) $v_{l, \text { eq }}^{k \text {,pure }}$ is the molar volume of pure component $k$ in equilibrium liquid state.

(xiv) $\gamma^{k}$ is the activity coefficient.

4.1. Equilibrium Equations. We will use the equilibrium equations for a multicomponent system in the following form:

$$
\begin{aligned}
& \int_{0}^{P}\left(v_{g}^{k}-v_{g}^{k, \text { pure }}\right) d P^{\prime}-\int_{0}^{P}\left(v_{l}^{k}-v_{l}^{k, \text { pure }}\right) d P^{\prime} \\
& \quad+R T \ln \frac{c_{g}^{k}}{c_{l}^{k}}+\int_{P_{*}^{\mathrm{I}, k}}^{P}\left(v_{g}^{k, \text { pure }}-v_{l}^{k, \text { pure }}\right) d P^{\prime}=0, \\
& V_{g}=V_{g}\left(P, T, N_{g}^{1}, \ldots, N_{g}^{n}\right) ; \\
& V_{l}=V_{l}\left(P, T, N_{l}^{1}, \ldots, N_{l}^{n}\right) ; \\
& \sum_{k=1}^{n} c_{g}^{k}=1, \\
& \sum_{k=1}^{n} c_{l}^{k}=1 .
\end{aligned}
$$

Equation (30a) results from the equivalence between chemical potentials; (30b) and (30c) are two EOS for pseudogas and pseudoliquid. The proof of (30a) is given in Appendix A.

The molar volumes $v_{i}^{k}$ are defined if EOS (30b) and (30c) are given; the individual parameters of pure components as their pseudocritical pressures $P_{*}^{\mathrm{II}, k}$ are the known values.

The consistency of system $((30 a)-(30 d))$ is ensured, if the consistency conditions ((13a) and (13b)) are verified for all pure components. We assume that all individual phase diagrams for pure components are well adjusted to experimental data by applying the technique described in Section 3.

System of $n$ equations (30a) contains $2 n$ variables: $2 n-$ 2 phase concentrations $c_{g}^{k}$ and $c_{l}^{k}$ (due to (30d)), pressure $P$, and temperature $T$. Such a system is unclosed and contains $n$ independent variables. We select them as $P, T$, and $n-2$ phase concentrations called independent. (This means, in particular, that the pressure in the equilibrium is not uniquely defined and can vary within the two-phase region.)

Depending on the closure relationships, we obtain three main problems related to multicomponent equilibria:

(i) Flash calculation: at given $P, T$, and total composition of the fluid, $\left\{C^{k}\right\}_{k=1}^{n-1}$, it is necessary to calculate the composition of both phases and the molar fraction of liquid phase.

In this case, the equilibrium relationships (30a) are completed with $n-1$ material balance equations:

$$
C^{k} \equiv c_{g}^{k} S_{g}+c_{l}^{k}\left(1-S_{g}\right), \quad k=1, \ldots, n-1,
$$

where $S_{g}$ is the gas molar fraction.
Then the system of $2 n-1$ equations (30a) and (31) contains $2 n-1$ unknown variables: $2 n-2$ phase concentrations and saturation $S_{g}$. Therefore it is closed.

(ii) Calculation of the bubble-point curve: at given $T$, we assume that only liquid phase exists, while the gas saturation tends to zero. This means that the total composition is equal to the liquid composition: $c_{l}^{k}=$ $C^{k}(k=1, \ldots, n-1)$, and the disappearing gas phase has some limit values of phase concentrations.

Then the system of $n$ equations (30a) contains $n$ unknown variables: $n-1$ limit concentrations $c_{g}^{k}$ and pressure $P$, which is called the bubble-pressure, $P_{\mathrm{bbl}}$. Its is clear that $P_{\mathrm{bbl}}$ is the function of the total composition and temperature.

(iii) Calculation of the dew-point curve: At given $T$, we assume that only gas phase exists. Then $c_{g}^{k}=C^{k}$ $(k=1, \ldots, n-1)$ and so on, similar to bubble point.

4.2. Equivalent form of the Equilibrium Equations through the Activity Coefficient. It is useful to represent the partial volumes of components in mixture through the individual volumes of pure components. This can be done by using the concept of ideal mixing, which assumes that the mixture volume is the sum of the volumes of the pure components: $V=\sum_{k=1}^{n} V^{k, \text { pure }}$, where $V^{k \text {,pure }}$ is the volume occupied by component $k$ in the pure state at the same $P, T$.

From this definition, it is possible to obtain the following property of an ideal phase $\alpha: v_{\alpha}^{k}=v_{\alpha}^{k, \text { pure }}$, for any component $k$ (the definitions of variables are given at the beginning of Section 4).

For nonideal mixture, we can introduce the excess in volume, $V^{e}$, which takes into account the nonideality:

$$
V=\sum_{k=1}^{n} V^{k, \text { pure }}+V^{e}
$$

(for an ideal mixture, $V^{e} \equiv 0$ ).

According to the definition of the Gibbs energy, volume $V^{e}$ is related with the excess in the Gibbs energy with respect to that of an ideal mixture, $G^{e}: V^{e}=\partial G^{e} / \partial P$. Then this gives the following result for molar volume from (32):

$$
v^{k}=v^{k, \text { pure }}+\frac{\partial}{\partial P}\left(\frac{\partial G^{e}}{\partial N^{k}}\right)=v^{k, \text { pure }}+R T \frac{\partial \ln \gamma^{k}}{\partial P},
$$

where we have introduced the new variable called the activity coefficient of component $k, \gamma^{k}$.

The value $\partial G^{e} / \partial N^{k}$ is the excess in the chemical potential, which is usually presented in the logarithmic form through $\gamma^{k}$.

Applying (33) to both phases, we obtain the following from (30a):

$$
\begin{aligned}
& R T \ln \left(\frac{\gamma_{g}^{k}}{\gamma_{l}^{k}}\right)+R T \ln \frac{c_{g}^{k}}{c_{l}^{k}}+\int_{P_{*}^{\mathrm{II}, k}}^{P}\left(v_{g}^{k, \text { pure }}-v_{l}^{k, \text { pure }}\right) d P^{\prime} \\
& \quad=0, \quad k=1, \ldots, n,
\end{aligned}
$$


where $\gamma_{g}^{k}$ and $\gamma_{l}^{k}$ are the activity coefficients of component $k$ in gas and liquid. We take into account the fact that both activity coefficients tend to 1 when pressure tends to zero.

Two versions of the equilibrium equations, (30a) and (34), are equivalent, but they assume different use of EOS for liquid and gas: for (30a), we should use EOS to determine the partial volumes, while for (34) the EOS should give us the activity coefficient. The activity coefficient may be also determined through several specific models as NRTL expression (Nonrandom Two-Liquid) [20]. The NRTL equation is one of the most popular and efficient methods of describing nonideal mixing. This model assumes that, even in multicomponent mixtures, the interactions between molecules can be described as two to two interactions. As a result, the model for multicomponent system contains only binary fitting parameters. The NRTL equation can efficiently represent behaviour of various mixtures, even those of a highly nonideal nature. For a mixture of $n$ components, the NRTL equation is (in terms of the excess in Gibbs energy)

$$
G^{e}=R T \sum_{i=1}^{n} N^{i} \frac{\sum_{j=1}^{n} c^{j} \tau_{j i} E_{j i}}{\sum_{j=1}^{n} c^{j} E_{j i}}
$$

Other parameters are

$$
\begin{aligned}
& \tau_{j i}=\frac{g_{j i}-g_{i i}}{R T}, \\
& E_{j i}=e^{-\sigma_{j i} \tau_{j i}}, \\
& \qquad\left(\sigma_{j i}=\sigma_{j i}, \tau_{j j}=0\right) .
\end{aligned}
$$

$\tau_{j i}$ and $\sigma_{j i}$ are dimensionless adjustable interaction parameters, $g_{i j}$ is the characteristic potential of $i-j$ interaction, and parameter $\sigma$ is related to the nonrandomness of molecule motion in the mixture which varies from about 0.20 to 0.47 .

4.3. Multicomponent Phase Diagrams. The expected behaviour of the phase diagrams of mixtures is easily revealed from the equilibrium equations (30a). Let us consider a twocomponent mixture (component 1 is light), which satisfies the condition of the ideal mixing both in liquid and in gas. Then $v_{g}^{k}=v_{g}^{k, \text { pure }}, v_{l}^{k}=v_{l}^{k, \text { pure }}$, and system (30a) becomes

$$
\underbrace{R T \ln \frac{c_{g}^{k}}{c_{l}^{k}}}_{A}+\underbrace{\int_{P_{*}^{\mathrm{II}, k}}^{P}\left(v_{g}^{k, \text { pure }}-v_{l}^{k, \text { pure }}\right) d P^{\prime}}_{B}=0 .
$$

Compared with the similar equation for pure components, (14), we see that the presence of new term $A$ disturbs the equilibrium. As explained for (14), the term $B$ represents graphically the sum of one negative area shown in Figure 3(a) and one positive area. Term $A$ is also positive for the light component and augments the contribution of positive areas. To equilibrate their role, the negative area must become higher than for the pure light component. This means that the equilibrium pressure of the mixture will be lower than that of the pure light component. Similarly, it is possible to show that it will be higher than the equilibrium pressure for the pure heavy component. As the equilibrium pressure of a mixture depends on the total concentrations $C^{1}$, then it will continuously vary between $P_{\text {eq }}^{2}$ and $P_{\text {eq }}^{1}$ when the total composition of mixture changes.

The use of the same ideal gas model for all gaseous individual components significantly reduces the number of parameters which need to be fitted. Let us verify that such a simplification does not lead to some nonphysical situations.

This case is illustrated in Figure 8 for a two-component fluid. The two red curves correspond to two EOS for two pure components in a pseudoliquid state, while the pseudogas state is described by the same EOS of ideal gas for both pure components (the blue curve). Component 1 is light.

Figures $8(\mathrm{a})$ and 8 (b) illustrate the graphical determination of the equilibrium pressures $P_{\text {eq }}^{1}$ and $P_{\text {eq }}^{2}$ for two pure components. Pressure $P_{\text {eq }}^{1}$ is determined in such a way that the yellow surface $A$ would be equal to the yellow surface $B$. Pressure $P_{\text {eq }}^{2}$ results from the equality between the yellow surfaces $C$ and $D$. The results of these constructions are

$$
\begin{gathered}
P_{\mathrm{eq}}^{2}<P_{\mathrm{eq}}^{1}, \\
v_{g, \text { eq }}^{2}>v_{g, \text { eq }}^{1}, \\
v_{l, \text { eq }}^{2}>v_{l, \text { eq }}^{1},
\end{gathered}
$$

which satisfy the physical meaning: indeed, the equilibrium pressure must be lower for the heavier component, while the equilibrium molar volumes must by lower for the light component.

Thus, the use of the ideal EOS for all pure components does not lead to any physical contradiction.

4.4. Equilibrium Equations for Ideal Mixing in Gas and Nonideal Mixing in Liquid. Let the mixing in the liquid phase be nonideal, while the gas is ideal. Then $v_{g}^{k}=v_{g}^{k \text {,pure }}$, or $\gamma_{g}^{k}=1$ for all $k$. From (34), we obtain the following equilibrium equations for $k=1, \ldots, n$ :

$$
\begin{aligned}
& \int_{P_{*}^{\mathrm{II}, k}}^{P}\left(v_{g}^{k, \text { pure }}-v_{l}^{k, \text { pure }}\right) d P^{\prime}+R T \ln \frac{c_{g}^{k}}{c_{l}^{k}}-\left.R T \ln \gamma_{l}^{k}\right|_{P} \\
& \quad=0 .
\end{aligned}
$$

Using (35), we obtain the expression of the activity coefficients through the phase concentrations:

$$
\begin{aligned}
\ln \gamma_{l}^{k} \equiv & \frac{1}{R T} \frac{\partial G_{l}^{e}}{\partial N_{l}^{k}} \\
= & \frac{\sum_{j=1}^{n} c_{l}^{j} \tau_{j k} E_{j k}}{\sum_{m=1}^{n} c_{l}^{m} E_{m k}} \\
& +\sum_{i=1}^{n} \frac{c_{l}^{i} E_{k i}}{\sum_{m=1}^{n} c_{l}^{m} E_{m i}}\left(\tau_{k i}-\frac{\sum_{j=1}^{n} c_{l}^{j} \tau_{j i} E_{j i}}{\sum_{m=1}^{n} c_{l}^{m} E_{m i}}\right) .
\end{aligned}
$$




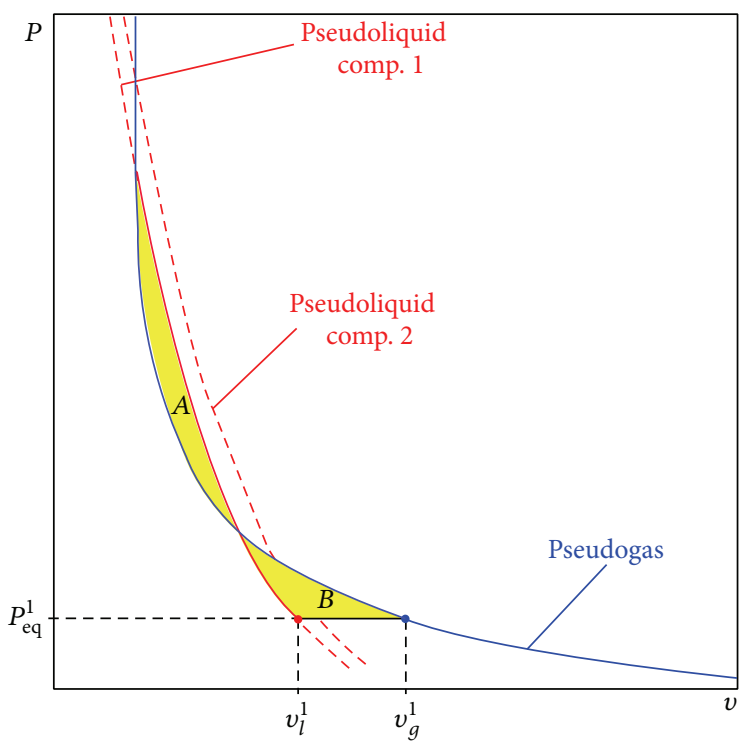

(a)

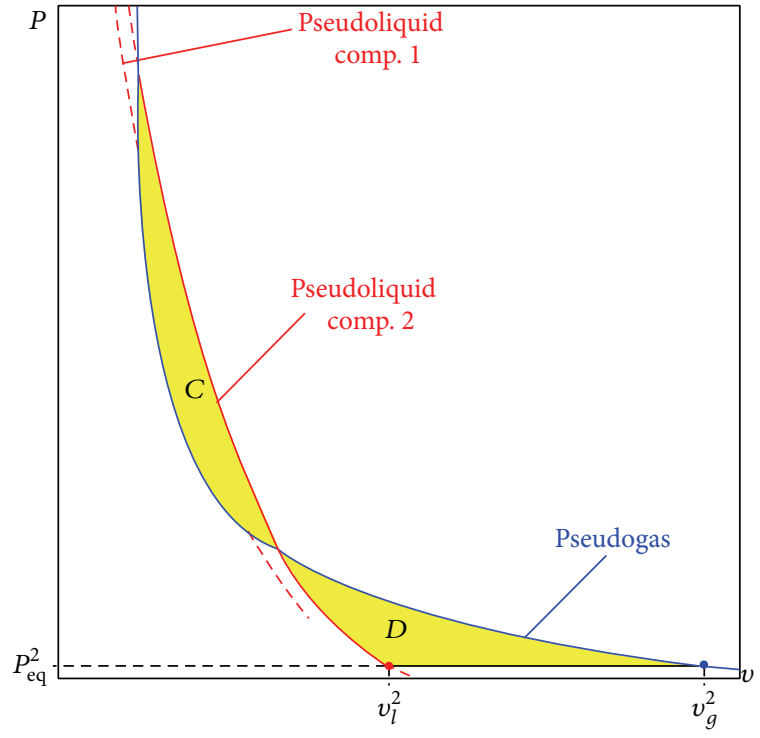

(b)

FIGURE 8: Example of consistency of the same EOS for gas for individual components: graphical determination of the equilibrium pressures $P_{\text {eq }}^{1}(\mathrm{a})$ and $P_{\text {eq }}^{2}(\mathrm{~b})$.

4.5. Equilibrium for Virial EOS for Gas and Exponential EOS for Liquid. Let the pure components be a liquid with exponential EOS and a gas described by virial EOS $(k=$ $1, \ldots, n)$ :

$$
\begin{aligned}
& v_{g}^{k, \text { pure }}=\frac{R T}{P}+v_{g 0}^{k}, \\
& v_{l}^{k, \text { pure }}=v_{l 0}^{k} e^{-\beta_{k}\left(P-P_{0}^{k}\right)},
\end{aligned}
$$

where $v_{g 0}^{k, \text { pure }}, v_{l 0}^{k}, \beta^{k}, P_{*}^{\mathrm{II}, k}$, and $P_{0}^{k}$ are the parameters of the model which determine the behaviour of the envelope of the phase diagram. They are selected to comply with experimental data on pure components and satisfy consistency conditions imposed by the envelope (Section 3 ). For the ideal gas $v_{g 0}^{k, \text { pure }} \equiv 0$.

The equilibrium equations (39) become $(k=1, \ldots, n)$

$$
\begin{gathered}
R T \ln \frac{c_{g}^{k}}{c_{l}^{k}}+v_{g 0}^{k}\left(P-P_{*}^{\mathrm{II}, k}\right)+R T \ln \frac{P}{P_{*}^{\mathrm{II}, k}}-R T \ln \gamma_{l}^{k} \\
+\frac{v_{l 0}^{k}}{\beta_{k}}\left[e^{-\beta_{k}\left(P-P_{0}^{k}\right)}-e^{-\beta_{k}\left(P_{*}^{\mathrm{II}, k}-P_{0}^{k}\right)}\right]=0 .
\end{gathered}
$$
form:

In dimensionless form these equations take the following

$$
\begin{aligned}
\ln \frac{c_{g}^{k}}{c_{l}^{k}} & +\alpha_{g k}\left(p-p_{*}^{k}\right)+\ln \frac{p}{p_{*}^{k}}-\ln \gamma_{l}^{k} \\
& +\alpha_{l k}\left[e^{-\varepsilon_{k}\left(p-\omega_{k}\right)}-e^{-\varepsilon_{k}\left(p_{*}^{k}-\omega_{k}\right)}\right]=0,
\end{aligned}
$$

where

$$
\begin{aligned}
\alpha_{g k} & \equiv \frac{v_{g 0}^{k} P_{0}^{k}}{R T}, \\
\alpha_{l k} & \equiv \frac{v_{l 0}^{k}}{\beta_{k} R T}, \\
\varepsilon_{k} & \equiv \beta_{k} P_{0}^{k}, \\
p_{*}^{k} & \equiv \frac{P_{*}^{\mathrm{II}, k}}{P_{0}^{1}}, \\
\omega_{k} & \equiv \frac{P_{0}^{k}}{P_{0}^{1}}, \\
p & \equiv \frac{P}{P_{0}^{1}} .
\end{aligned}
$$

4.6. Analytical Solutions for Phase Concentrations in TwoComponent Case. For a binary mixture, the equilibrium equations (43) take the following form:

$$
\begin{aligned}
& \ln \frac{c_{g}^{1}}{c_{l}^{1}}+\alpha_{g 1}\left(p-p_{*}^{1}\right)+\ln \frac{p}{p_{*}^{1}} \\
& \quad+\alpha_{l 1}\left[e^{-\varepsilon_{1}(p-1)}-e^{-\varepsilon_{1}\left(p_{*}^{1}-1\right)}\right]-\ln \gamma_{l}^{1}=0, \\
& \ln \frac{1-c_{g}^{1}}{1-c_{l}^{1}}+\alpha_{g 2}\left(p-p_{*}^{2}\right)+\ln \frac{p}{p_{*}^{2}} \\
& \quad+\alpha_{l 2}\left[e^{-\varepsilon_{2}\left(p-\omega_{2}\right)}-e^{-\varepsilon_{2}\left(p_{*}^{2}-\omega_{2}\right)}\right]-\ln \gamma_{l}^{2}=0,
\end{aligned}
$$

where the superscript 2 means the number of components but not "square." 
System (45) determines two concentrations, $c_{g}^{1}$ and $c_{g}^{2}$, as the functions of pressure $p$.

This system has an explicit analytical solution for phase concentrations $c_{g}^{1}(P, T)$ and $c_{l}^{1}(P, T)$ in two cases, (1) the ideal mixing in both phases and (2) the moderately nonideal mixing in the liquid phase:

$$
\begin{aligned}
& c_{g}^{1}(p, T)=\frac{1-K_{2}}{1-K_{2} / K_{1}}, \\
& c_{l}^{1}(p, T)=\frac{1-K_{2}}{K_{1}-K_{2}},
\end{aligned}
$$

where $K$-values are defined as $K_{k} \equiv c_{g}^{k} / c_{l}^{k}$. For ideal mixing in both phases, $K$-values are calculated directly from (45) (for $k=1,2)$ :

$$
\begin{aligned}
K_{k} & =\frac{p_{*}^{k}}{p} \exp \left[-\alpha_{g k}\left(p-p_{*}^{k}\right)\right. \\
& \left.-\alpha_{l k}\left(e^{-\varepsilon_{k}\left(p-\omega_{k}\right)}-e^{-\varepsilon_{k}\left(p_{*}^{k}-\omega_{k}\right)}\right)\right] .
\end{aligned}
$$

For nonideal mixing in liquid, let us assume the hypothesis of moderate nonideality: the interactions between different molecules and identical molecules are nearly equal: $g_{12} \simeq g_{21} \simeq g_{11} \simeq g_{22}$, which leads to $\tau_{j i} \simeq 0$. Then $K$-values in (46) are calculated as

$$
\begin{aligned}
K_{k} & =\frac{p_{*}^{k}}{p}\left(1+\ln \gamma_{0}^{k}\right) \exp \left[-\alpha_{g k}\left(p-p_{*}^{k}\right)\right. \\
& \left.-\alpha_{l k}\left(e^{-\varepsilon_{k}\left(p-\omega_{k}\right)}-e^{-\varepsilon_{k}\left(p_{*}^{k}-\omega_{k}\right)}\right)\right],
\end{aligned}
$$

where

$$
\begin{aligned}
& \ln \gamma_{0}^{1} \\
& \quad=\left(c_{l 0}^{2}\right)^{2}\left[\tau_{21}\left(\frac{E_{21}}{c_{l 0}^{1}+c_{l 0}^{2} E_{21}}\right)^{2}+\frac{\tau_{12} E_{12}}{\left(c_{l 0}^{2}+c_{l 0}^{1} E_{12}\right)^{2}}\right], \\
& \ln \gamma_{0}^{2} \\
& \quad=\left(c_{l 0}^{1}\right)^{2}\left[\tau_{12}\left(\frac{E_{12}}{c_{l 0}^{2}+c_{l 0}^{1} E_{12}}\right)^{2}+\frac{\tau_{21} E_{21}}{\left(c_{l 0}^{1}+c_{l 0}^{2} E_{21}\right)^{2}}\right], \\
& c_{l 0}^{1}=\frac{1-K_{02}}{K_{01}-K_{02}}, \\
& c_{l 0}^{2}=1-c_{l 0}^{1} .
\end{aligned}
$$

Coefficients $K_{01}$ and $K_{02}$ are calculated by using formulae (47). This solution is developed in Appendix B.

4.7. Analytical Solutions for Phase Concentrations in ThreeComponent Case. In a three-component system, three equations of phase equilibrium contain four phase concentrations $c_{g}^{1}, c_{g}^{2}, c_{l}^{1}$, and $c_{l}^{2}$, which means one concentration is independent. We will select $c_{l}^{1}$ as this independent parameter. Then the equilibrium system determines three other concentrations as the functions of the equilibrium pressure, temperature, and $c_{l}^{1}$. The dimensionless equilibrium equations are

$$
\begin{aligned}
& \ln \frac{c_{g}^{1}}{c_{l}^{1}}+\alpha_{g 1}\left(p-p_{*}^{1}\right)+\ln \frac{p}{p_{*}^{1}} \\
& +\alpha_{l 1}\left[e^{-\varepsilon^{1}(p-1)}-e^{-\varepsilon_{1}\left(p_{*}^{1}-1\right)}\right]=\ln \gamma_{l 1}, \\
& \ln \frac{c_{g}^{2}}{c_{l}^{2}}+\alpha_{g 2}\left(p-p_{*}^{2}\right)+\ln \frac{p}{p_{*}^{2}} \\
& +\alpha_{l 2}\left[e^{-\varepsilon_{2}\left(p-\omega_{2}\right)}-e^{-\varepsilon_{2}\left(p_{*}^{2}-\omega_{2}\right)}\right]=\ln \gamma_{l 2}, \\
& \ln \frac{1-c_{g}^{1}-c_{g}^{2}}{1-c_{l}^{1}-c_{l}^{2}}+\alpha_{g 3}\left(p-p_{*}^{3}\right)+\ln \frac{p}{p_{*}^{3}} \\
& +\alpha_{l 3}\left[e^{-\varepsilon_{3}\left(p-\omega_{3}\right)}-e^{-\varepsilon_{3}\left(p_{*}^{3}-\omega_{3}\right)}\right]=\ln \gamma_{l 3} .
\end{aligned}
$$

As said previously, this system has an explicit analytical solution regarding phase concentrations in two cases, (i) ideal mixing everywhere and (ii) moderately nonideal mixing in liquid:

$$
\begin{aligned}
& c_{g}^{1}\left(p, T, c_{l}^{1}\right)=K_{1} c_{l}^{1}, \\
& c_{l}^{2}\left(p, T, c_{l}^{1}\right)=\frac{1-K_{3}}{K_{2}-K_{3}}-\frac{K_{1}-K_{3}}{K_{2}-K_{3}} c_{l}^{1}, \\
& c_{g}^{2}\left(p, T, c_{l}^{1}\right)=K_{2}\left(\frac{1-K_{3}}{K_{2}-K_{3}}-\frac{K_{1}-K_{3}}{K_{2}-K_{3}} c_{l}^{1}\right) .
\end{aligned}
$$

For ideal mixing in both phases, the following was obtained for $K$-values $(k=1,2,3)$ :

$$
\begin{aligned}
K_{k} & =\frac{p_{*}^{k}}{p} \exp \left[-\alpha_{g k}\left(p-p_{*}^{k}\right)\right. \\
& \left.-\alpha_{l k}\left(e^{-\varepsilon_{i}\left(p-\omega_{k}\right)}-e^{-\varepsilon_{i}\left(p_{*}^{k}-\omega_{k}\right)}\right)\right] .
\end{aligned}
$$

For nonideal mixing in liquid,

$$
\begin{aligned}
K_{k} & =\frac{p_{*}^{k}}{p}\left(1+\ln \gamma_{l 0}^{k}\right) \exp \left[-\alpha_{g k}\left(p-p_{*}^{k}\right)\right. \\
& \left.-\alpha_{l k}\left(e^{-\varepsilon_{k}\left(p-\omega_{k}\right)}-e^{-\varepsilon_{k}\left(p_{*}^{k}-\omega_{k}\right)}\right)\right],
\end{aligned}
$$

where $\gamma_{l 0}^{k}$ is calculated through (40) in which one should use $c_{l 0}^{q}$ instead of $c_{l}^{q}$, for all $q ; c_{g 0}^{k}, c_{l 0}^{k}$, and $K_{k 0}$ are calculated as for ideal mixing by using formulae (51) and (52).

As mentioned in Section 4.1, the equilibrium equations can be completed with $n$ material balance equations (31), for given total composition of the fluid $C^{k}$. This makes it possible to determine parameter $c_{l}^{1}$ considered as independent in the equilibrium equations:

$$
\begin{aligned}
& C^{1}=c_{l}^{1}\left[1+\left(K_{1}-1\right) S_{g}\right], \\
& C^{2}=\left[\frac{1-K_{3}}{K_{2}-K_{3}}-\frac{K_{1}-K_{3}}{K_{2}-K_{3}} c_{l}^{1}\right]\left[1+\left(K_{2}-1\right) S_{g}\right],
\end{aligned}
$$


TABLE 1: Parameters of dissymmetric models for pure components used to calculate binary mixtures.

\begin{tabular}{lcccccc}
\hline & $\begin{array}{c}T \\
\mathrm{~K}\end{array}$ & $\begin{array}{c}P_{\mathrm{eq}} \\
\text { bar } \\
\text { (calculated by PR EOS) }\end{array}$ & $\begin{array}{c}P_{0} \\
\text { bar }\end{array}$ & $\begin{array}{c}v_{l^{3}} \\
10^{-6} \mathrm{~m}^{3} / \mathrm{mol}\end{array}$ & $\begin{array}{c}P_{*}^{\text {II }} \\
\text { bar }\end{array}$ & $\begin{array}{c}\beta \\
10^{-4} \text { bar }^{-1}\end{array}$ \\
\hline 2-Methylpentane, $\mathrm{C}_{6} \mathrm{H}_{14}$ & 328.15 & 0.809 & 60.7 & 129.9 & 6565.0 & 5.292 \\
Heptane, $\mathrm{C}_{7} \mathrm{H}_{16}$ & 328.15 & 0.232 & 54.8 & 149.7 & 6832.1 & 5.347 \\
Octane, $\mathrm{C}_{8} \mathrm{H}_{18}$ & 328.15 & 0.089 & 49.8 & 169.5 & 6792.8 & 5.550 \\
Pentane, $\mathrm{C}_{5} \mathrm{H}_{12}$ & 328.15 & 1.580 & 67.4 & 109.4 & 7357.9 & 5.143 \\
Benzene, $\mathrm{C}_{6} \mathrm{H}_{6}$ & 328.15 & 0.380 & 97.52 & 85.5 & 13550 & 2.786 \\
\hline Butane, $\mathrm{C}_{4} \mathrm{H}_{10}$ & 377.6 & 16.82 & 76 & 123.3 & 3992.4 & 7.028 \\
Decane, $\mathrm{C}_{10} \mathrm{H}_{22}$ & 377.6 & 0.117 & 42.2 & 219.6 & 5721.1 & 6.497 \\
\hline
\end{tabular}

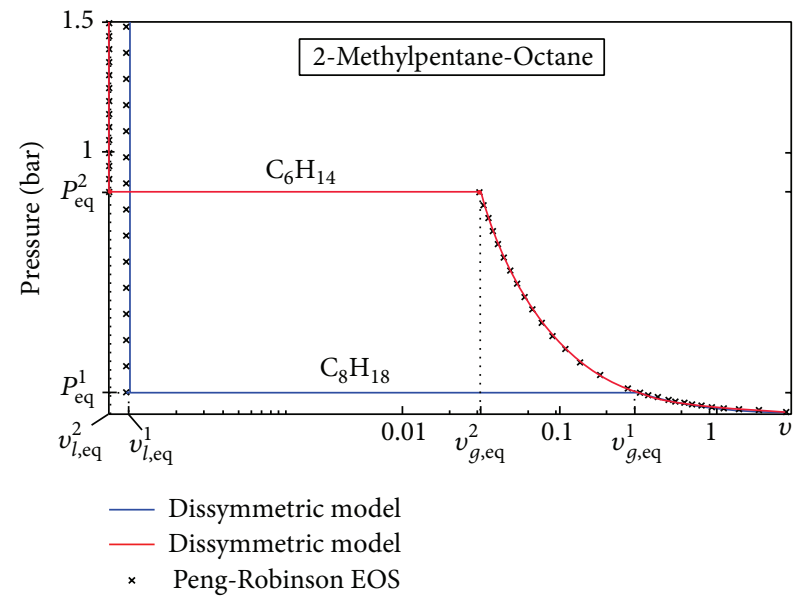

(a)

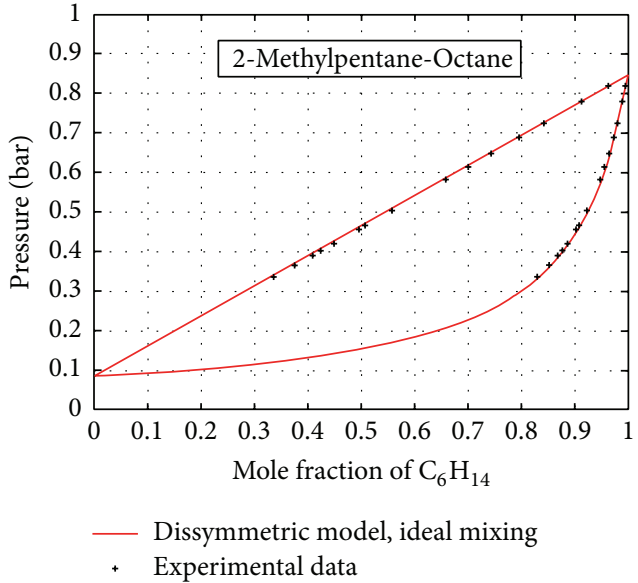

(b)

Figure 9: Binary phase diagrams for 2-Methylpentane $\left(\mathrm{C}_{6} \mathrm{H}_{14}\right)$-Octane $\left(\mathrm{C}_{8} \mathrm{H}_{18}\right)$ at $T=328.15 \mathrm{~K}$.

where $S_{g}$ is the gas molar fraction. We obtain two equations with two unknown variables: $c_{l}^{1}$ and $S_{g}$.

The envelopes of the two-phase domain are calculated by using the same equations in which $c_{l}^{1}=C^{1}$ for the bubble curve (transition to single-phase liquid) and $c_{l}^{1}=C^{1} / K_{1}$ for the dew curve (transition to single-phase gas), which results from (54) if $S_{g}=0$ and $S_{g}=1$, respectively.

\section{Comparison with Experimental Data and Cubic EOS}

5.1. Two-Component Case. The gas-liquid equilibrium for a two-component mixture at constant temperature is usually represented by diagrams " $P$ versus $C^{1}$," where $C^{1}$ is the total concentration of the lightest component. At high $C^{1}$, the system is gaseous; at high $P$, it is liquid, while a two-phase domain exists between these two states.

The dissymmetric model of pure components was presented by exponential EOS for liquid and ideal gas.

We used the experimental data for several binary mixtures published in [21]:

(i) 2-Methylpentane $\left(\mathrm{C}_{6} \mathrm{H}_{14}\right)$ and Heptane $\left(\mathrm{C}_{7} \mathrm{H}_{16}\right)$.

(ii) 2-Methylpentane and Octane $\left(\mathrm{C}_{8} \mathrm{H}_{18}\right)$. (iii) Heptane and Octane.

(iv) Butane $\left(\mathrm{C}_{4} \mathrm{H}_{10}\right)$ and Decane $\left(\mathrm{C}_{10} \mathrm{H}_{22}\right)$.

(v) Pentane $\left(\mathrm{C}_{5} \mathrm{H}_{12}\right)$ and Benzene $\left(\mathrm{C}_{6} \mathrm{H}_{6}\right)$.

Parameters of the dissymmetric models of pure components obtained by fitting their $P-v$ curves with the diagrams calculated by using Peng-Robinson EOS are presented in Table 1.

For mixture, the phase diagrams (the envelops of the twophase zone) were constructed by using the model presented in this paper in terms of $P$ versus the molar volume $v$ (see Figure 9(a)) and $P$ versus the overall concentration of the light component $C^{1}$ (Figure 9(b)). Diagram $P-v$ was compared with the Peng-Robinson model, as the experimental data on such diagrams practically do not exist. For diagrams $P-C^{1}$, we used experimental data.

The comparison of calculations by using the dissymmetric model with experimental data for Butane $\left(\mathrm{C}_{4} \mathrm{H}_{10}\right)$-Decane $\left(\mathrm{C}_{10} \mathrm{H}_{22}\right)$ mixture is presented in Figure 10.

The case of nonideal mixing is illustrated in Figure 11 for the mixture Pentane-Benzene, for which there exist experimental data. 


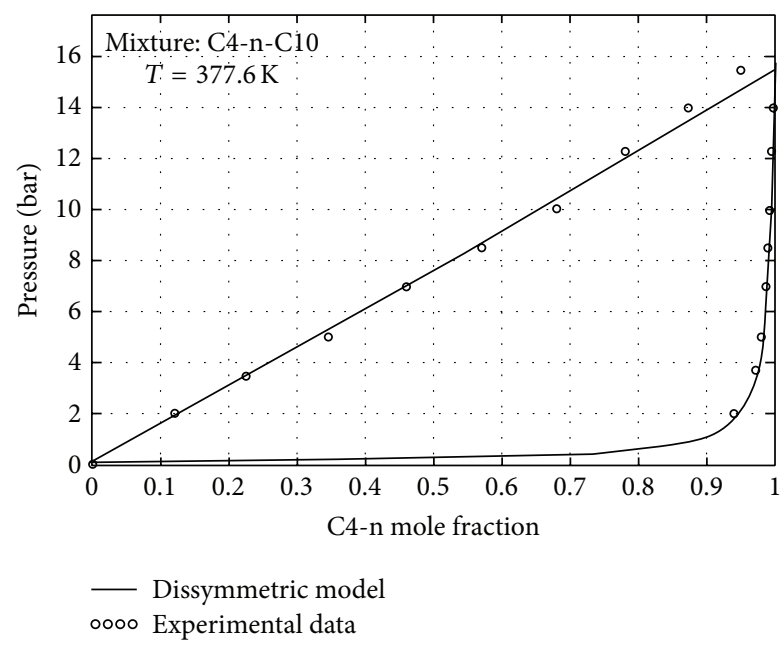

Figure 10: Binary phase diagrams for Butane $\left(\mathrm{C}_{4} \mathrm{H}_{10}\right)$-Decane $\left(\mathrm{C}_{10} \mathrm{H}_{22}\right)$ mixture at $T=377.6 \mathrm{~K}$.

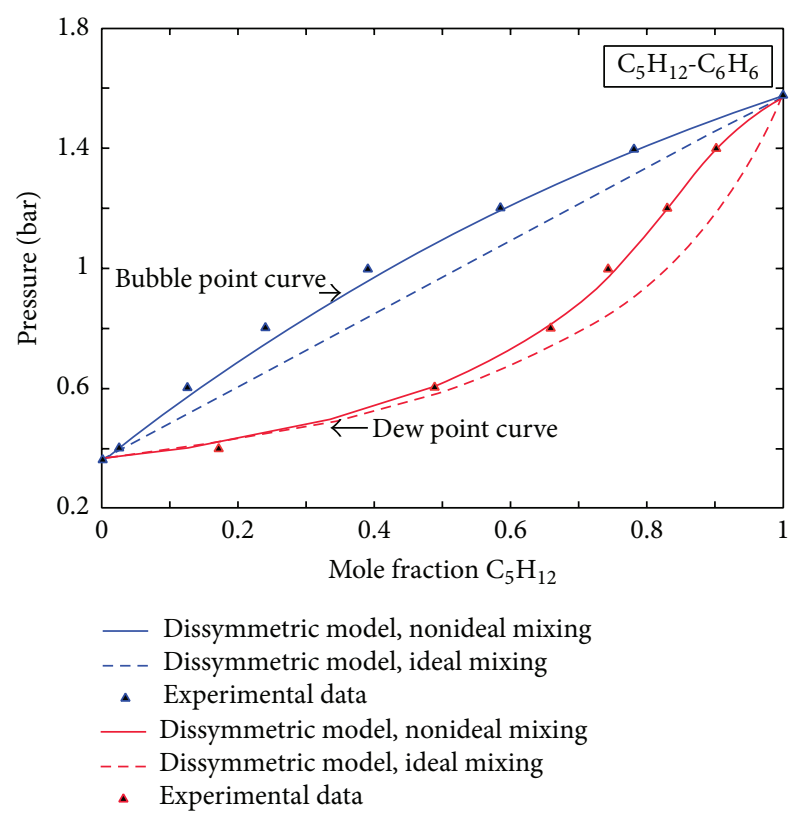

FIgURE 11: Binary phase diagrams for Pentane $\left(\mathrm{C}_{5} \mathrm{H}_{12}\right)$-Benzene $\left(\mathrm{C}_{6} \mathrm{H}_{6}\right)$ mixture at $T=328.15 \mathrm{~K}$.

Two other cases of comparison with experimental data are shown in Figure 17 for Heptane and Octane (a) and for 2-Methylpentane and Heptane (b).

As seen, the nonideal dissymmetric model gives satisfactory results with respect to real data.

We underline that, for mixtures, only the parameters of pure components should be fit.

5.2. Three-Component Case. The gas-liquid equilibrium of a three-component mixture at constant temperature and pressure is often represented in an equilateral triangle (ternary diagram). Its height is equal to unity. A mixture composition is represented by a point within the triangle, so that the molar fractions of three components are given by three heights lowered from that point on three sides.

For each total composition $\left(C^{1}, C^{2}, C^{3}\right)$, phase concentrations can be calculated from (51)-(54). We drew the bubble curve and the dew curve by using the following relationships resulting from (51)-(54):

(i) For bubble point curve,

$$
\begin{aligned}
& C^{1}=a_{1}+a_{2} C^{2}, \\
& a_{1}=\frac{K_{3}-1}{K_{3}-K_{1}}, \\
& a_{2}=\frac{K_{2}-K_{3}}{K_{3}-1} .
\end{aligned}
$$

(ii) For dew point curve,

$$
C^{1}=K_{1}\left(a_{1}+a_{2} C^{2}\right) .
$$

We have calculated the ternary diagrams for several mixtures by using the dissymmetric model and compared them to experimental data existing in the literature or to other methods of calculation. Parameters of the dissymmetric model obtained by fitting the phase equilibrium of pure components are presented in Table 2.

The first three Figures 12, 13, and 14 illustrate the comparison of the results obtained by the dissymmetric model with simulations based on Peng-Robinson EOS. The dew point curve is blue, and the bubble point curves are red. The tie lines are dashed.

Four other Figures 15 and 16 show the comparison of the results obtained by the dissymmetric model with experimental data.

Remark. To use the dissymmetric model, it is necessary to fit only the parameters of pure components, by using the procedure described in Section 3. The consecutive calculation of phase composition is performed by analytical formulae. Therefore, once the dissymmetric model has been adjusted for pure components, it calculates the multicomponent equilibrium instantaneously.

The time of adjustment for a pure component by using algorithm presented in Section 3 is lower than $2 \mathrm{~s}$ (for various processors), if the equilibrium pressure and volumes are known (they can be found in various source data).

\section{Application to Capillary Condensation}

6.1. Equilibrium Equations in Presence of Capillary Pressure. In the situation when the interface between gas and liquid is curvilinear, the equilibrium gas and liquid pressures are different due to the capillary pressure calculated according to the Laplace equation:

$$
\begin{aligned}
& P_{g}=P_{l}+P_{c}, \\
& P_{c}=\frac{2 \sigma}{r},
\end{aligned}
$$

where $P_{c}$ is the capillary pressure, $\sigma$ is the surface tension, and $r$ is the mean curvature radius of the gas-liquid interface. 
TABLE 2: Parameters of fitting pure components used to calculate ternary mixtures.

\begin{tabular}{|c|c|c|c|c|c|c|}
\hline & $\begin{array}{l}T \\
\mathrm{~K}\end{array}$ & $\begin{array}{c}P_{\mathrm{eq}} \\
\text { bar } \\
\text { (calculated by PR EOS) }\end{array}$ & $\begin{array}{l}P_{0} \\
\text { bar }\end{array}$ & $\begin{array}{c}v_{l 0} \\
10^{-6} \mathrm{~m}^{3} / \mathrm{mol}\end{array}$ & $\begin{array}{l}P_{*}^{\mathrm{II}} \\
\text { bar }\end{array}$ & $\begin{array}{c}\beta \\
10^{-4} \operatorname{bar}^{-1}\end{array}$ \\
\hline Propane, $\mathrm{C}_{3} \mathrm{H}_{8}$ & 300 & 9.870 & 84 & 84.4 & 5726.2 & 5.253 \\
\hline Pentane, $\mathrm{C}_{5} \mathrm{H}_{12}$ & 300 & 0.732 & 67.4 & 109.4 & 7357.9 & 4.765 \\
\hline Decane, $\mathrm{C}_{10} \mathrm{H}_{22}$ & 300 & 0.0021 & 42.2 & 206.7 & 7510.2 & 5.531 \\
\hline Hydrogen Sulfide, $\mathrm{H}_{2} \mathrm{~S}$ & 290 & 14.155 & 180.08 & 37.5 & 13919 & 2.238 \\
\hline Propane, $\mathrm{C}_{3} \mathrm{H}_{8}$ & 290 & 7.600 & 84.02 & 81.2 & 6183 & 4.977 \\
\hline Pentane, $\mathrm{C}_{5} \mathrm{H}_{12}$ & 290 & 0.501 & 67.40 & 107.8 & 7669 & 4.650 \\
\hline Argon, Ar & 112 & 16.517 & 67.92 & 42.41 & 32847 & 8.410 \\
\hline Nitrogen, $\mathrm{N}_{2}$ & 112 & 7.582 & 97.20 & 28.14 & 7072.7 & 4.390 \\
\hline Methane, $\mathrm{CH}_{4}$ & 112 & 1.048 & 91.60 & 32.77 & 8915.3 & 3.905 \\
\hline $\mathrm{R} 22, \mathrm{CHF}_{2} \mathrm{Cl}$ & 273.2 & 4.29 & 99.8 & 63.83 & 8594 & 3.61 \\
\hline Nitrogen, $\mathrm{N}_{2}$ & 273.2 & - & 732.71 & 47.44 & 194.1 & 16.76 \\
\hline Carbon Dioxide, $\mathrm{CO}_{2}$ & 273.2 & 34.82 & 147.55 & 46.33 & 7498.3 & 3.71 \\
\hline $\mathrm{R} 30, \mathrm{CH}_{2} \mathrm{Cl}_{2}$ & 318.15 & 1.266 & 126 & 63.35 & 1359.9 & 2.585 \\
\hline $\mathrm{R} 10, \mathrm{CCl}_{4}$ & 318.15 & 0.351 & 91.2 & 92.37 & 1077.5 & 3.395 \\
\hline $\mathrm{R} 20, \mathrm{CHCl}_{3}$ & 318.15 & 0.567 & 107.4 & 76.34 & 1247 & 2.898 \\
\hline $\mathrm{R} 142 \mathrm{~b}, \mathrm{CH}_{3}-\mathrm{CF}_{2} \mathrm{Cl}$ & 323.25 & 6.647 & 81.1 & 91.96 & 6278 & 4.949 \\
\hline $\mathrm{R} 140 \mathrm{a}, \mathrm{CCl}_{3}-\mathrm{CH}_{3}$ & 323.25 & 0.1481 & 102.8 & 87.09 & 13244 & 2.861 \\
\hline $\mathrm{R} 141 \mathrm{~b}, \mathrm{CH}_{3}-\mathrm{CFCl}_{2}$ & 323.25 & 1.8055 & 83.88 & 92.46 & 8314 & 4.074 \\
\hline
\end{tabular}

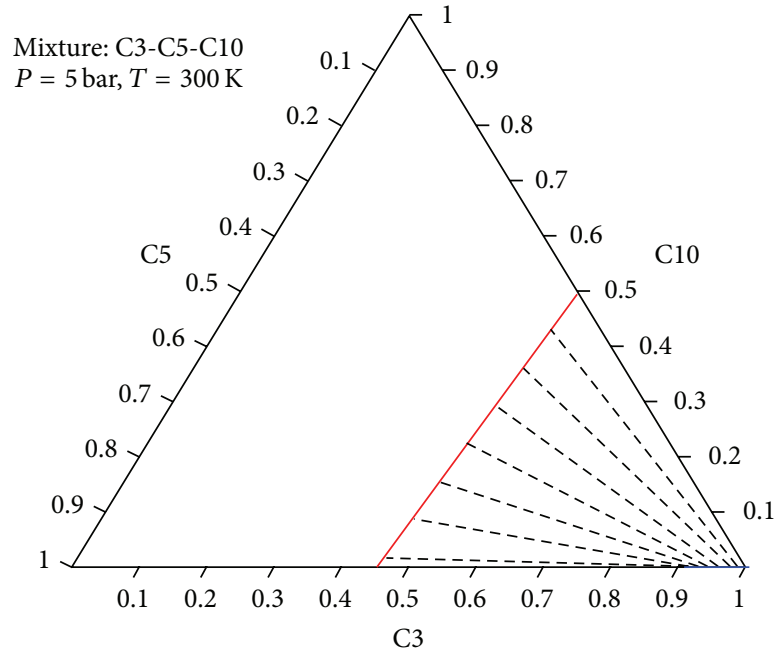

(a)

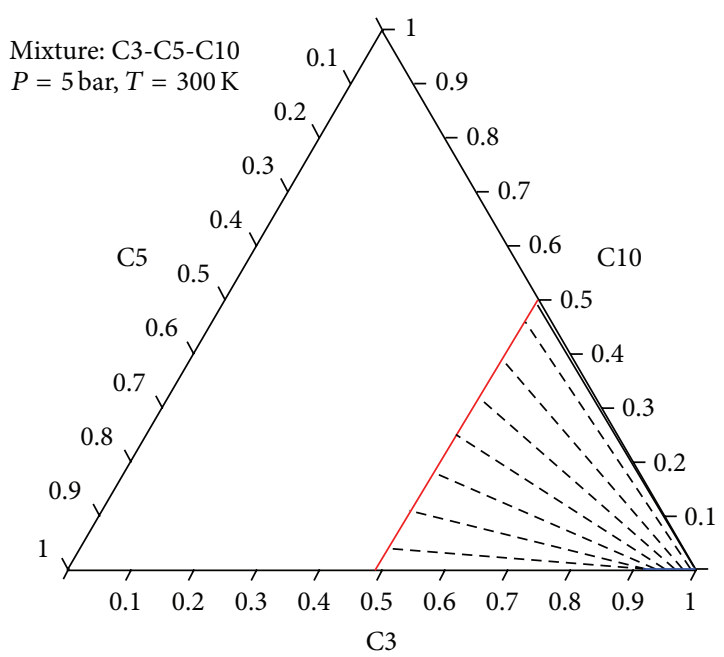

(b)

FIgURE 12: Ternary phase diagram for Propane-Pentane-Decane mixture, for $P=5$ bar and $T=300 \mathrm{~K}$ : (a) the dissymmetric model and (b) Peng-Robinson EOS.

The general equilibrium equations ((30a)-(30d)) for multicomponent mixtures become

$$
\begin{aligned}
& \int_{0}^{P}\left(v_{g}^{k}-v_{g}^{k, \text { pure }}\right) d P^{\prime}-\int_{0}^{P-P_{c}}\left(v_{l}^{k}-v_{l}^{k, \text { pure }}\right) d P^{\prime} \\
& \quad+R T \ln \frac{c_{g}^{k}}{c_{l}^{k}}+\int_{P_{*}^{\mathrm{II}, k}}^{P} v_{g}^{k, \text { pure }} d P^{\prime}-\int_{P_{*}^{\mathrm{II}, k}}^{P-P_{c}} v_{l}^{k, \text { pure }} d P^{\prime} \\
& \quad=0, \quad k=1, \ldots, n,
\end{aligned}
$$

where $P$ is the gas pressure.
For virial EOS for gas and exponential EOS for liquid, we obtain, similar to (43),

$$
\begin{aligned}
\ln \frac{c_{g}^{k}}{c_{l}^{k}} & +\alpha_{g k}\left(p-p_{*}^{k}\right)+\ln \frac{p}{p_{*}^{k}}-\ln \gamma_{l}^{k} \\
& +\alpha_{l k}\left[e^{-\varepsilon_{k}\left(p-C a-\omega_{k}\right)}-e^{-\varepsilon_{k}\left(p_{*}^{k}-\omega_{k}\right)}\right]=0,
\end{aligned}
$$

where $\mathrm{Ca} \equiv P_{c} / P_{0}^{1}$ is the capillary number. 


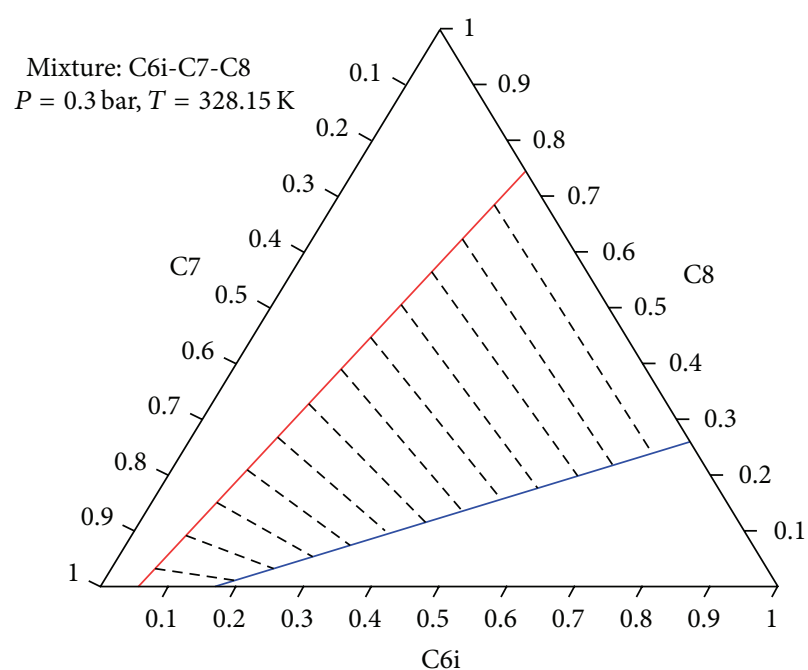

(a)

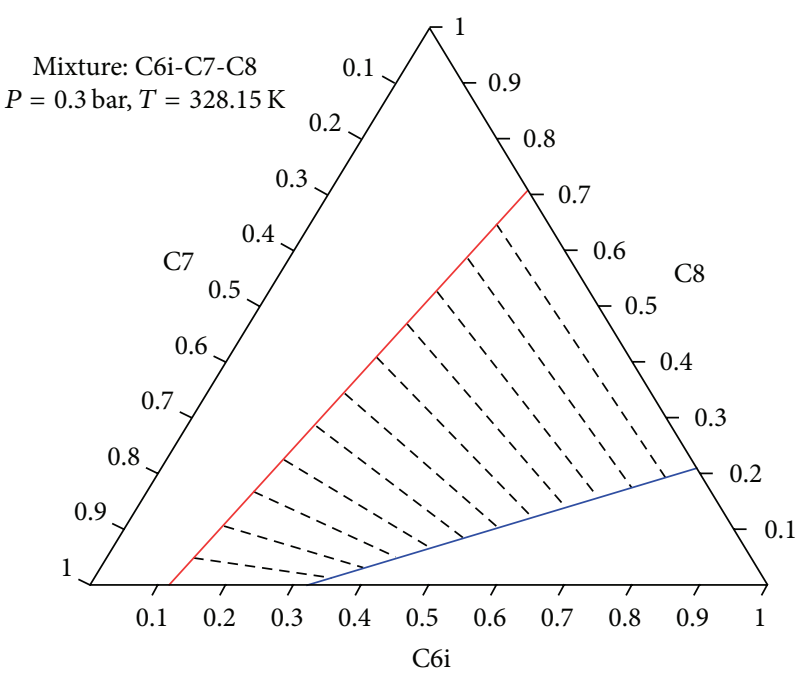

(b)

Figure 13: Ternary phase diagram for 2-Methylpentane-Heptane-Octane mixture, for $P=0.3$ bar and $T=328.15 \mathrm{~K}$ : (a) the dissymmetric model and (b) Peng-Robinson EOS.

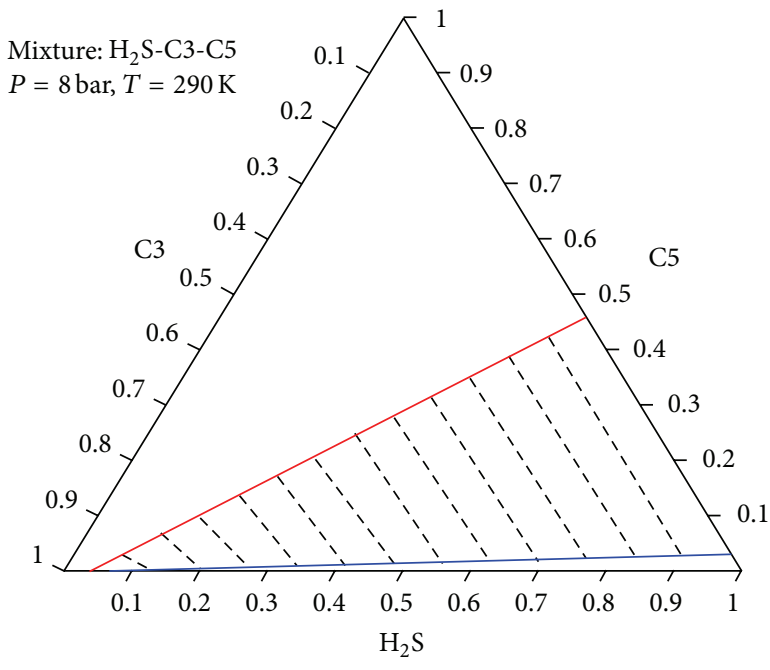

(a)

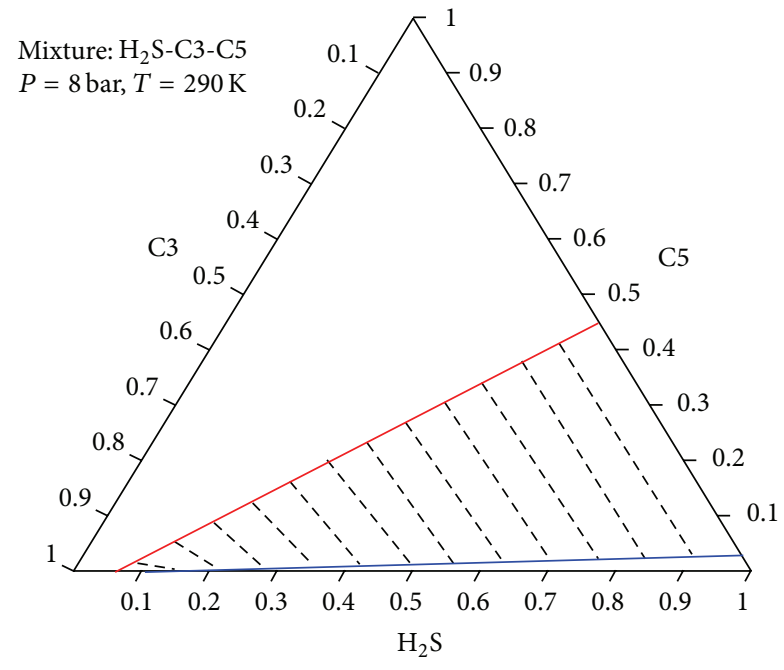

(b)

Figure 14: Ternary phase diagram for Hydrogen Sulfide $\left(\mathrm{H}_{2} \mathrm{~S}\right)$-Propane $\left(\mathrm{C}_{3} \mathrm{H}_{8}\right)$-Pentane $\left(\mathrm{C}_{5} \mathrm{H}_{12}\right)$, for $P=8$ bar and $\mathrm{T}=290 \mathrm{~K}$ : $(\mathrm{a})$ the dissymmetric model and (b) the Peng-Robinson EOS.

6.2. Calculation of the Phase Composition at Nonzero Capillary Pressure. For ternary mixture, the following can be obtained for $K$-values $(k=1,2,3)$, similar to (52):

$$
\begin{aligned}
K_{k} & =\frac{p_{*}^{k}}{p}\left(1+\ln \gamma_{l 0}^{k}\right) \exp \left[-\alpha_{g k}\left(p-p_{*}^{k}\right)\right. \\
& \left.-\alpha_{l k}\left(e^{-\varepsilon_{k}\left(p-\mathrm{Ca}-\omega_{k}\right)}-e^{-\varepsilon_{k}\left(p_{*}^{k}-\omega_{k}\right)}\right)\right] .
\end{aligned}
$$

Then the phase concentrations are calculated by using the same formulae (51).

Parameter $\gamma_{l 0}^{k}$ is zero for ideal mixture. For nonideal solution, it is calculated through (40), in which one should use $c_{l 0}^{q}$ instead of $c_{l}^{q}$, for all $q ; c_{g 0}^{k}, c_{l 0}^{k}$, and $K_{k 0}$ are calculated by using formulae (51) but with $K$-values corresponding to ideal mixing, that is, by using (60), in which $\gamma_{l 0}^{k}=0$.

The examples of calculation of the phase diagrams at nonzero capillary pressure are given in Figure 17.

The values of $P_{c}$ we have used are proper to natural porous media: for pure components the curvature radius of gasliquid interface is assumed to be of the order of pore radius $\left(\sim 200 A^{\circ}\right)$, and the surface tension of each component was calculated using the Brock and Bird Model. The capillary pressure of the mixture was estimated as the mean value $\left(P_{c} \approx\right.$ $\left.0.5\left(P_{c 1}+P_{c 2}\right)\right)$. 


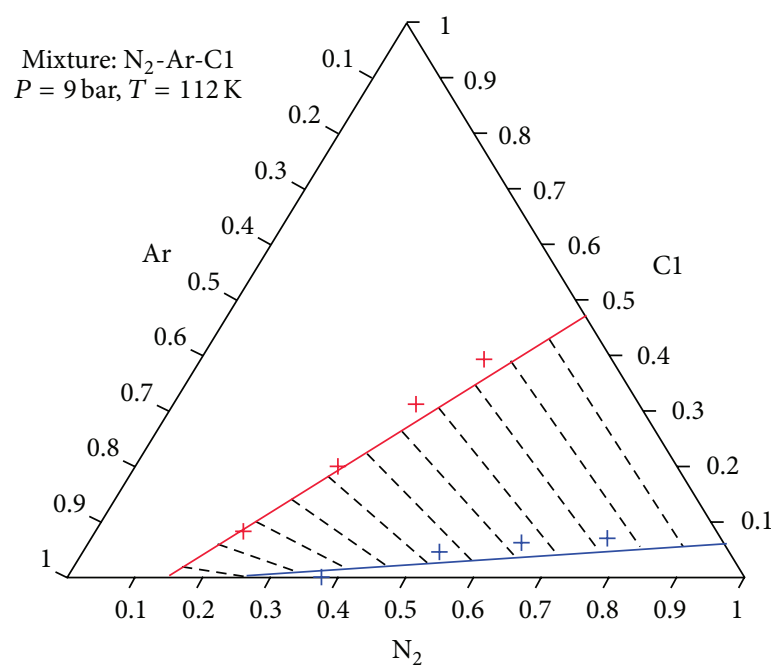

(a)

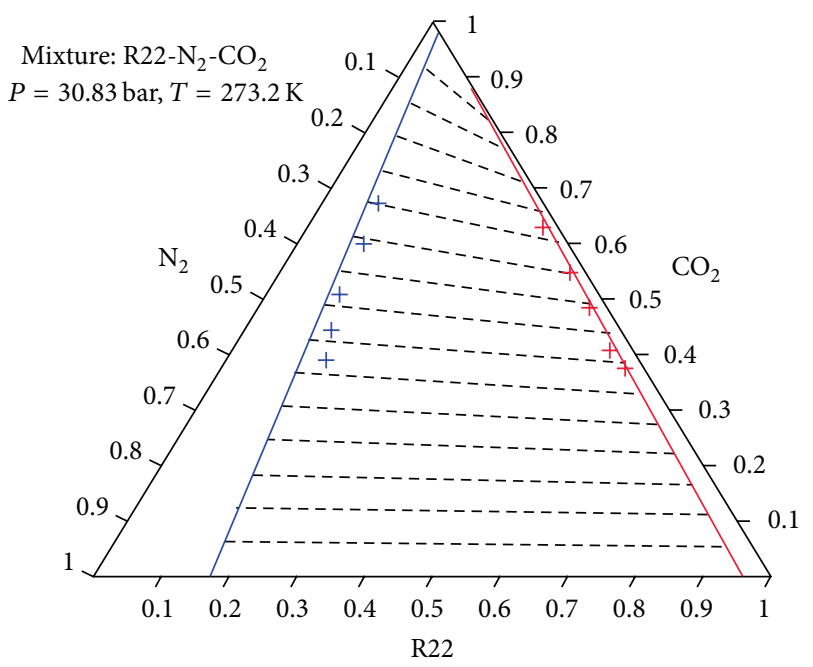

(b)

FIGURE 15: Ternary phase diagrams calculated by the dissymmetric model (lines) and experimental data (crosslets): (a) mixture of Nitrogen $\left(\mathrm{N}_{2}\right)$-Argon (Ar)-Methane $\left(\mathrm{CH}_{4}\right)$ at $T=112 \mathrm{~K}$ and $P=9$ bar; experimental data from [16]; (b) mixture of Chlorodifluoromethane (R22)Nitrogen $\left(\mathrm{N}_{2}\right)$-Carbon Dioxide $\left(\mathrm{CO}_{2}\right)$ at $T=273.2 \mathrm{~K}$ and $P=30.83$ bar; experimental data from [17].

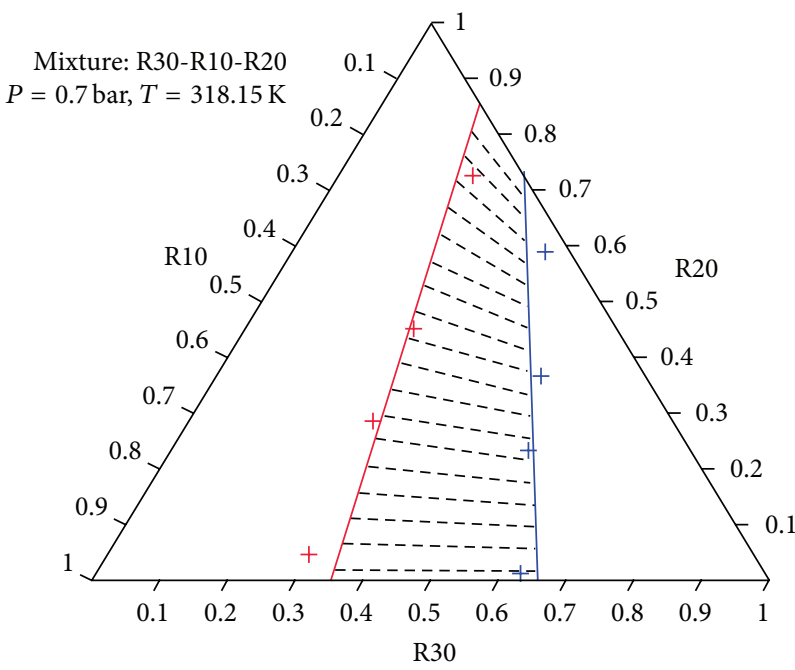

(a)

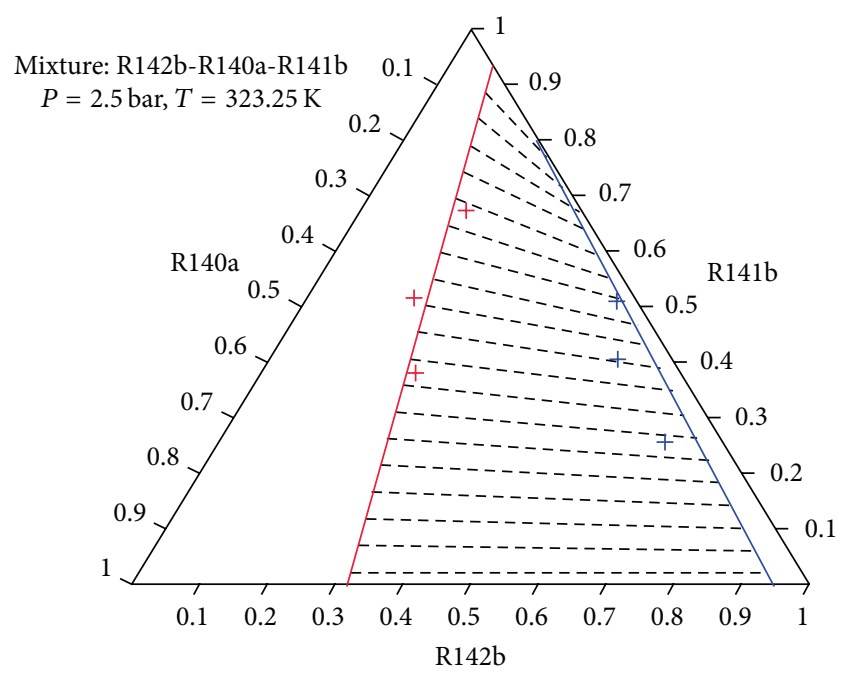

(b)

Figure 16: Ternary phase diagrams calculated by the dissymmetric model (lines) and experimental data (crosslets): (a) mixture of Dichloromethane (R30)-Tetrachloromethane (R10)-Trichloromethane (R20) at $T=318.15 \mathrm{~K}$ and $P=0.7$ bar; experimental data from [18]; (b) mixture of Chlorodifluoromethane (R142b)-1,1,1-Trichloroethane (R140a)-1,1-Dichloro-1-fluoroethane (R141b) at $T=323.5 \mathrm{~K}$ and $P=2.5$ bar; experimental data from [19].

6.3. Kelvin's Equation of Capillary Condensation for OneComponent Case. For one-component fluid, the equilibrium equations (58) determine a unique equilibrium pressure in gas phase $P_{\text {eq }}$ :

$$
\begin{aligned}
& \int_{P_{*}^{\mathrm{II}}}^{P_{\mathrm{eq}}}\left(v_{g}(P, T)-v_{l}(P, T)\right) d P+\int_{P_{\mathrm{eq}}-P_{c}}^{P_{\mathrm{eq}}} v_{l}(P, T) d P \\
& \quad=0 .
\end{aligned}
$$

At given EOS for pseudogas, $v_{g}=v_{g}(P, T)$, and for pseudoliquid $v_{l}=v_{l}(P, T)$, (61) represents a nonlinear equation with respect to the equilibrium pressure $P_{\text {eq }}$.

Due to the presence of the new term (with respect to (10)), in this case consistency can be reached even if we have only one point of intersection between the EOS for pseudoliquid and pseudogas. This for instance is the case of incompressible liquid and ideal gas, for which the vertical line $v_{l}=$ const intersects the hyperbolic curve of the ideal gas only at one 


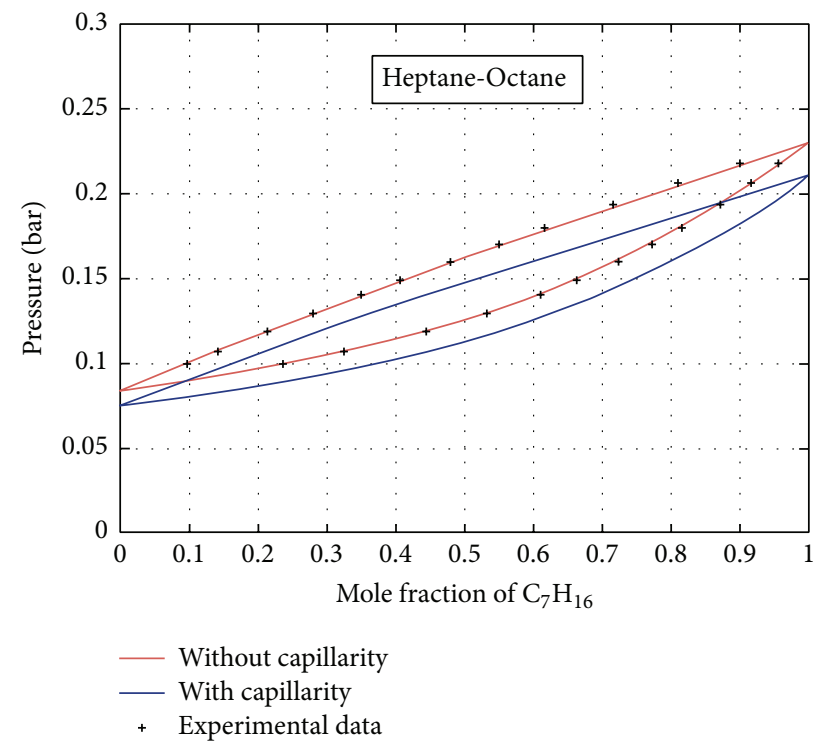

(a)

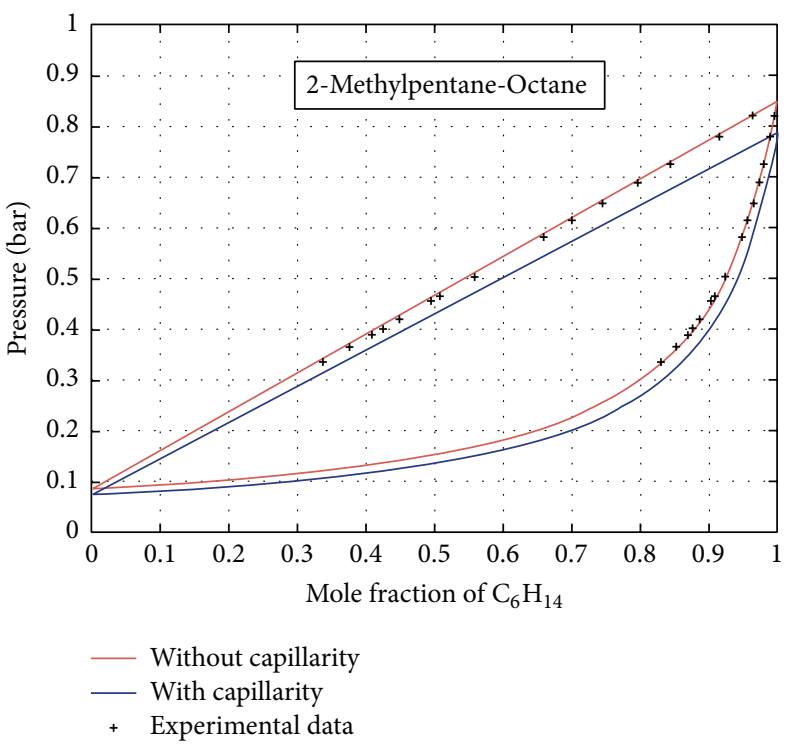

(b)

Figure 17: Binary phase diagrams for Heptane $\left(\mathrm{C}_{7} \mathrm{H}_{16}\right)$-Octane $\left(\mathrm{C}_{8} \mathrm{H}_{18}\right)(\mathrm{a})$ and 2-Methylpentane $\left(\mathrm{C}_{6} \mathrm{H}_{14}\right)$-Octane (b) with capillary pressure and without it at $T=328.15 \mathrm{~K}$.

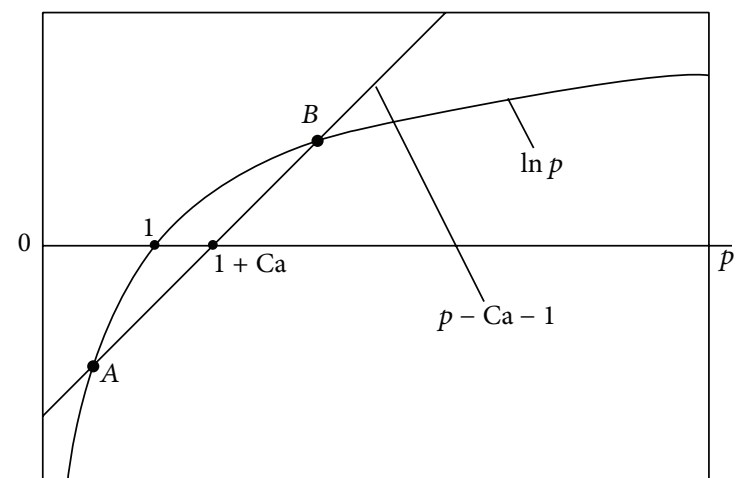

FIGURE 18: Graphical interpretation of (62). The physical nontrivial solution corresponds to point $A$.

point $P_{*}^{\mathrm{I}}: v_{l}=R T / P_{*}^{\mathrm{I}}$. Then we obtain the following from (61) by integrating: $R T \ln \left(P_{\text {eq }} / P_{*}^{\mathrm{I}}\right)=v_{l}\left(P_{\text {eq }}-P_{c}-P_{*}^{\mathrm{I}}\right)$. This yields

$$
\ln p=p-\mathrm{Ca}-1,
$$

where $p \equiv P_{\text {eq }} / P_{*}^{\mathrm{I}}$; the capillary number $\mathrm{Ca} \equiv 2 \sigma /\left(r P_{*}^{\mathrm{I}}\right)$.

This equation has two nontrivial solutions, as shown in Figure 18, where the left- and right-hand sides of (62) are presented. They correspond to points $A$ and $B$ (point $p=1$ on the horizontal axis corresponds to $P=P_{*}^{\mathrm{I}}$ ).

Point $B$ has no physical meaning, as the corresponding value $p_{B}>1$; then $P_{\text {eq }}>P_{*}^{\mathrm{I}}$. This means that the molar volume of the coexisting liquid is higher than that of gas $\left(v_{\mathrm{g} \text {,eq }}=R T / P_{\mathrm{eq}}\right)$, which is physically impossible. So the true solution corresponds to point $A$. We obtain the characteristic pressure $P_{A}$ which is lower than $P_{*}^{\mathrm{I}}$, at which the true gas and liquid can coexist.
According to Kelvin, for a plan gas-liquid interface, the capillary pressure is zero; therefore (62) yields $\ln p=p-1$, which has a unique solution $p=1$, or $P_{\mathrm{eq}}=P_{*}^{\mathrm{I}}$. Hence, we can consider pressure $P_{*}^{\mathrm{I}}$ to be the pressure of gas-liquid coexistence at zero capillarity. It should also be noted again here that the case of $P_{c}=0$ cannot be described by the model of incompressible liquid and ideal gas.

When the gas pressure is close to $P_{*}^{\mathrm{I}}$, or $p \rightarrow 1$ (i.e., the capillarity is sufficiently low), then (62) gives $\ln p \approx-\mathrm{Ca}$, or

$$
\frac{P_{\mathrm{eq}}}{P_{*}^{\mathrm{I}}} \approx \exp \left(\frac{2 \sigma}{r P_{*}^{\mathrm{I}}}\right)=\exp \left(\frac{2 v_{l} \sigma}{r R T}\right)
$$

which is the Kelvin equation for the capillary condensation which relates the pressure in gas under a curved gas-liquid interface with the pressure at a plane interface (strictly speaking, the exponential function in (63) should be expanded into the Taylor series).

The case of positive Ca corresponds to a gas bubble in continuous liquid or to a porous medium wetted more by liquid than by gas. The opposite case corresponds to $\mathrm{Ca}<0$. However, (62) has no solutions if $\mathrm{Ca}<0$, as in this case the straight line $p-\mathrm{Ca}-1$ is placed under the logarithmic curve in Figure 18. This means that the liquid drops in continuum gas cannot exist in the equilibrium and are therefore unstable.

\section{Conclusion}

As shown, the development of the idea of using different EOS for gas and liquid required us to develop a mathematical tool and to introduce some new concepts such as a pseudogas, pseudoliquid, and pseudocritical point and the consistency conditions. The consistency conditions, which determine the solvability of the equilibrium equations based on two 
different EOS for gas and liquid, are the main elements of this theory which determine its novelty. We have obtained exact theoretical equations which determine the pseudocritical pressure (6) and the equilibrium pressure (10). For the case of the ideal gas and the exponential EOS for liquid, they have the form (17) and (19), respectively.

In Section 5, we have presented the mathematical technique of using the relationships (17) and (19) to determine the adjustable parameters of two EOS for pure components.

In particular cases, we have shown that the model of the ideal gas for all pure components is consistent and can be used to calculate gas-liquid equilibrium, while the model of incompressible liquid is nonconsistent.

We have proven that the consistency condition means graphically that two curves $P(v)$ for gas and liquid cross each other at two points $\left(P_{*}^{\mathrm{I}}\right.$ and $\left.P_{*}^{\mathrm{II}}\right)$ and the area between these curves located between points $P_{*}^{\mathrm{I}}$ and $P_{*}^{\mathrm{II}}$ must be equivalent to the area between points $P_{\text {eq }}$ and $P_{*}^{\mathrm{I}}$, as seen in Figure 3(a). This graphical construction determines the equilibrium pressure $P_{\mathrm{eq}}$ and represents the analogue of the Maxwell equal area rule but turned counterclockwise.

The extension of this approach to multicomponent case leads to a more general system of equilibrium equations, which can be formulated through partial volumes (30a) or through activity coefficient (34). It was obtained from the fundamental relationships for chemical potentials through the molar volumes. This model does not require any additional adjustment, except the adjustments already done for each individual pure component. We have illustrated the developed approach using the example of ideal mixing in gas and nonideal mixing in liquid. For individual pure components, we used the virial EOS for gas or ideal gas and the exponentially compressible liquid, which satisfy the conditions of self-consistency. We have shown that a dissymmetric model is capable of ensuring explicit analytical relationships for phase concentrations.

In the last section we extended this approach to the equilibrium in presence of capillary forces, which leads to the extension of Kelvin's formula of capillary condensation to multicomponent case.

The objective of the present paper was to illustrate the feasibility of the suggested approach in principle. The comparison to some experimental data and simulations with Peng-Robinson EOS has shown that the developed approach is fundamentally able to predict the equilibrium of twocomponent and three-component mixtures. We used the data for various kinds of components: hydrocarbons (C1C10), aromatic hydrocarbons (benzene), Oxygen, Nitrogen, Carbon Dioxide, Argon, Hydrogen Sulfide, fluorines, and chlorines. For all these data the results calculated by analytical formulae for phase concentrations are in satisfactory agreement with experimental data, even by using the simplest types of EOS. Obviously, this approach can be improved and advanced through the use of more complex EOS.

The practical advantage of using the developed theory, rather than that based on a uniform EOS for both phases, consists of the fact that this approach highly simplifies the flush calculations by leading to explicit analytical relationships for phase concentrations even for multicomponent nonideal mixtures instead of traditional nonlinear time-consuming algorithms. One efficient application of this approach would be in the field of oil engineering as currently the simulation of the multiphase compositional fluid flow is hindered by the time it takes to carry out the necessary thermodynamic calculations.

\section{Appendices}

\section{A. Proof of (30a)}

(1.) The following relationship for the chemical potential of component $k$ in an arbitrary phase through the chemical potential of the ideal gas is known in classical thermodynamics [1-4]:

$$
\begin{aligned}
& \mu^{k}\left(P, T, c^{1}, \ldots, c^{n}\right) \\
& =\int_{0}^{P}\left[v^{k}\left(\bar{P}, T, c^{1}, \ldots, c^{n}\right)-\frac{R T}{\bar{P}}\right] d \bar{P} \\
& \quad+\mu^{k, \text { ideal }}\left(P, T, c^{k}\right), \\
& \mu^{k, \text { ideal }}\left(P, T, c^{k}\right)=R T \ln \frac{P c^{k}}{P_{r}^{k}}+\mu^{k, \text { pure }}\left(P_{r}^{k}, T\right),
\end{aligned}
$$

where $P_{r}^{k}$ is an arbitrary reference value of pressure which may be different for various components. Then

$$
\begin{aligned}
\mu^{k}= & \int_{0}^{P}\left[v^{k}-\frac{R T}{\bar{P}}\right] d \bar{P}+R T \ln \frac{P c^{k}}{P_{r}^{k}} \\
& +\mu^{k, \text { ideal }} \text { pure }\left(P_{r}^{k}, T\right) .
\end{aligned}
$$

(2.) For a pure component we obtain

$$
\begin{aligned}
\mu^{k, \text { pure }}(P, T)= & \int_{0}^{P}\left[v^{k, \text { pure }}(\bar{P}, T)-\frac{R T}{\bar{P}}\right] d \bar{P} \\
& +R T \ln \frac{P}{P_{r}^{k}}+\mu^{k \text {, ideal }} \text { pure }\left(P_{r}^{k}, T\right) .
\end{aligned}
$$

(3.) Let us subtract these two relationships from each other:

$$
\mu^{k}-\mu^{k, \text { pure }}=\int_{0}^{P}\left[v^{k}-v^{k, \text { pure }}\right] d \bar{P}
$$

(4.) Using (5), we obtain

$$
\begin{aligned}
\mu^{k}= & \int_{0}^{P}\left(v^{k}-v^{k, \text { pure }}\right) d \bar{P}+R T \ln c^{k}+\int_{P_{r}^{k}}^{P} v^{k, \text { pure }} d \bar{P} \\
& +\mu^{k, \text { pure }}\left(P_{r}^{k}, T\right) .
\end{aligned}
$$


(5.) Substituting the last relationship in the general equilibrium equation, $\mu_{g}^{k}\left(P_{g}, T, c_{g}^{1}, \ldots, c_{g}^{n}\right)=\mu_{l}^{k}\left(P_{l}, T, c_{l}^{1}, \ldots, c_{l}^{n}\right)$, we obtain

$$
\begin{gathered}
\int_{0}^{P_{g}}\left(v_{g}^{k}-v_{g}^{k, \text { pure }}\right) d P-\int_{0}^{P_{l}}\left(v_{l}-v_{l}^{k, \text { pure }}\right) d P \\
\quad+R T \ln \frac{c_{g}^{k}}{c_{l}^{k}}+\int_{P_{r}^{k}}^{P_{g}} v_{g}^{k, \text { pure }} d P-\int_{P_{r}^{k}}^{P_{l}} v_{l}^{k, \text { pure }} d P \\
+\mu_{g}^{k, \text { pure }}\left(P_{r}^{k}, T\right)-\mu_{l}^{k, \text { pure }}\left(P_{r}^{k}, T\right)=0 .
\end{gathered}
$$

(6.) Selecting the reference pressures as the second pseudocritical pressures of individual components, $P_{r}^{k}=P_{*}^{\mathrm{II}, k}$, we obtain (30a), because $\mu_{g}^{k \text {,pure }}\left(P_{*}^{\mathrm{II}, k}, T\right)=\mu_{l}^{k, \text { pure }}\left(P_{*}^{\mathrm{II}, k}, T\right)$ at the point where the difference between the pressure, temperature, and molar volumes of pseudogas and pseudoliquid disappears.

\section{B. Proof of (47) and (48)}

Let us assume that $\delta \equiv \max \left\{\left|\tau_{i j}\right|\right\}$ is a small parameter. Then one represents the solution in the following form:

$$
\begin{aligned}
c_{\alpha}^{k} & =c_{\alpha 0}^{k}+c_{\alpha 1}^{k}, \quad \alpha=g, l, \\
K^{k} & =K_{0}^{k}+K_{1}^{k},
\end{aligned}
$$

where $c_{\alpha 0}^{k}$ and $K_{k 0}=c_{g 0}^{k} / c_{l 0}^{k}$ are the values which correspond to the ideal mixing and $c_{\alpha 1}^{k}$ and $K_{k 1}$ are the small deviations of order $\delta$.

All the values $\ln \gamma_{l}^{k}$ are of order $\delta$ and disappear in zero approximation. Substituting these expressions in (43) and applying the method of perturbation, one obtains the following for the zero approximation:

$$
\begin{aligned}
\ln K_{k 0} & +\alpha_{g k}\left(p-p_{*}^{k}\right)+\ln \frac{p}{p_{*}^{k}} \\
+ & \alpha_{l k}\left[e^{-\varepsilon_{k}\left(p-\omega_{k}\right)}-e^{-\varepsilon_{k}\left(p_{*}^{k}-\omega_{k}\right)}\right]=0,
\end{aligned}
$$

And, for the terms of order $\delta$,

$$
\frac{K_{k 1}}{K_{k 0}}-\ln \gamma_{l 0}^{k}=0,
$$

where $\ln \gamma_{l 0}^{k}$ is defined through (40) in which $c_{\alpha}^{k}=c_{\alpha 0}^{k}(\alpha=$ $g, l)$.

Relationships (B.2) give (47) for $K_{0}^{k}$, while (B.1) and (B.3) yield $K_{k}=K_{k 0}\left(1+\ln \gamma_{l 0}^{k}\right)$, which leads to (48).

\section{Conflict of Interests}

The authors declare that there is no conflict of interests regarding the publication of this paper.

\section{Acknowledgments}

The research was partially supported by the Groupement de Recherche MOMAS: Modélisation Mathématique et Simulations Numériques liées aux études d’entreposage souterrain de déchets radioactifs (CNRS, ANDRA, EDF, CEA, BRGM).

\section{References}

[1] A. I. Brusilovsky, Phase Transition Under Exploitation of Oil and Gas Reservoirs, Graal, Moscow, Russia, 2002 (Russian).

[2] A. Firoozabadi, Thermodynamics of Hydrocarbon Reservoirs, McGraw-Hill, New York, NY, USA, 1999.

[3] M. Hillert, Phase Equilibria, Phase Diagrams and Phase Transformations, Cambridge University Press, Cambridge, UK, 2007.

[4] S. M. Walas, Phase Equilibria in Chemical Engineering, Elsevier, Butterworth, Malaysia, 1985.

[5] M. L. Michelsen, "The isothermal flash problem. Part I. Stability," Fluid Phase Equilibria, vol. 9, no. 1, pp. 1-19, 1982.

[6] M. L. Michelsen, "The isothermal flash problem. Part II: phase split calculation," Fluid Phase Equilibria, vol. 9, no. 1, pp. 21-40, 1982.

[7] M. L. Michelsen, "Simplified flash calculations for cubic equations of state," Industrial \& Engineering Chemistry Process Design and Development, vol. 25, no. 1, pp. 184-188, 1986.

[8] B. H. Jensen and A. Fredenslund, "A simplified flash procedure for multicomponent mixtures containing hydrocarbons and one non-hydrocarbon using two-parameter cubic equations of state," Industrial and Engineering Chemistry Research, vol. 26, no. 10, pp. 2129-2134, 1987.

[9] Y. Li and R. T. Johns, "Rapid flash calculations for compositional simulation," SPE Reservoir Evaluation \& Engineering, vol. 9, no. 5, pp. 521-529, 2006.

[10] S. Oladyshkin and M. Panfilov, "Limit thermodynamic model for compositional gas-liquid systems moving in a porous medium," Transport in Porous Media, vol. 70, no. 2, pp. 147-165, 2007.

[11] S. Oladyshkin, S. Skachkov, I. Panfilova, and M. Panfilov, "Upscaling fractured media and streamline HT-splitting in compositional reservoir simulation," Oil \& Gas Science and Technology, vol. 62, no. 2, pp. 137-146, 2007.

[12] A. P. Plres, P. G. Bedrikovetsky, and A. A. Shapiro, "Analytical modeling for two-phase EOR processes: splitting between hydrodynamics and thermodynamics," in Proceedings of the SPE Annual Technical Conference and Exhibition, SPE Paper 89919 MS, pp. 573-583, Houston, Tex, USA, September 2004.

[13] A. V. Koldoba and E. V. Koldoba, "Model equation of state and Gibbs energy for numerical modeling of the problems of multicomponent flow with phase transition in porous media," Geochemistry, vol. 4, pp. 573-576, 2004 (Russian).

[14] A. A. Shapiro and E. H. Stenby, "Kelvin equation for a non-ideal multicomponent mixture," Fluid Phase Equilibria, vol. 134, no. 1-2, pp. 87-101, 1997.

[15] H. Orbey and S. I. Sandler, Modeling Vapor-Liquid Equilibria: Cubic Equations of State and Their Mixing Rules, Cambridge University Press, Cambridge, UK, 1998.

[16] R. C. Miller, A. J. Kidnay, and M. J. Hiza, "Liquid-vapor equilibria at $112.00 \mathrm{~K}$ for systems containing nitrogen, argon, and methane," AIChE Journal, vol. 19, no. 1, pp. 145-151, 1973. 
[17] X. Wang, Y. Wang, J. Shi, and B. C.-Y. Lu, "Isothermal vaporliquid equilibria at elevated pressures for the systems containing nitrogen, carbon dioxide, and chlorodifluoromethane," Journal of Chemical and Engineering Data, vol. 36, no. 4, pp. 436-439, 1991.

[18] G. Rulewicz, H. Schuberth, and E. Leibnitz, "Untersuchungen über das Dampf-Flüssigkeits-Phasengleichgewicht des Systems Methylenchlorid/Chloroform/Tetrachlorkohlenstoff bei $45^{\circ} \mathrm{C}$ unter Verwendung einer gaschromatographischen Analysenmethode," Journal für Praktische Chemie, vol. 37, no. 3-4, pp. 122-136, 1968.

[19] Y. W. Kang and Y. Y. Lee, "Vapor-liquid equilibria for the systems composed of 1-chloro-1,1-difluoroethane, 1,1-dichloro1-fluoroethane, and 1,1,1-trichloroethane at $50.1^{\circ} \mathrm{C}$," Journal of Chemical and Engineering Data, vol. 41, no. 2, pp. 303-305, 1996.

[20] H. Renon and J. M. Prausnitz, "Local compositions in thermodynamic excess functions for liquid mixtures," AIChE Journal, vol. 14, no. 1, pp. 135-144, 1968.

[21] J. Gmehling, U. Onken, and W. Arlt, Chemistry Data Series: Vapor-Liquid Equilibrium Data Collection, Dechema, 1977. 

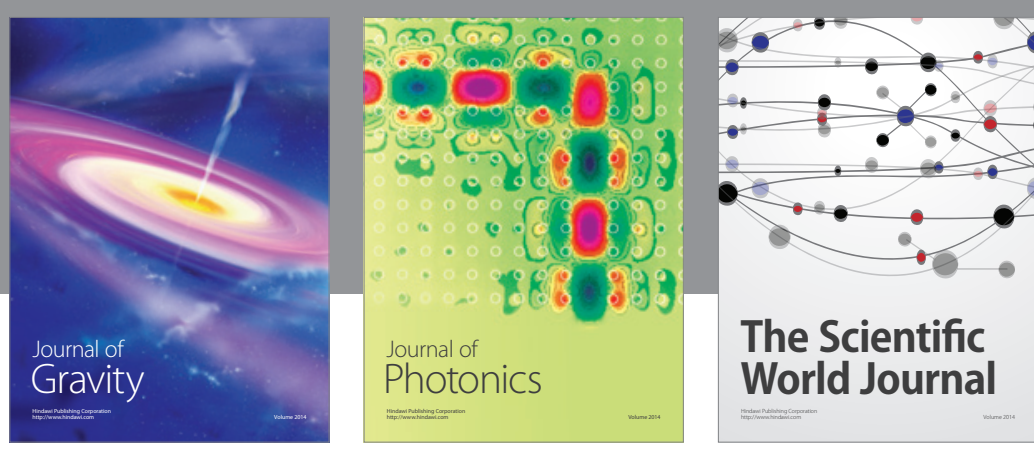

The Scientific World Journal
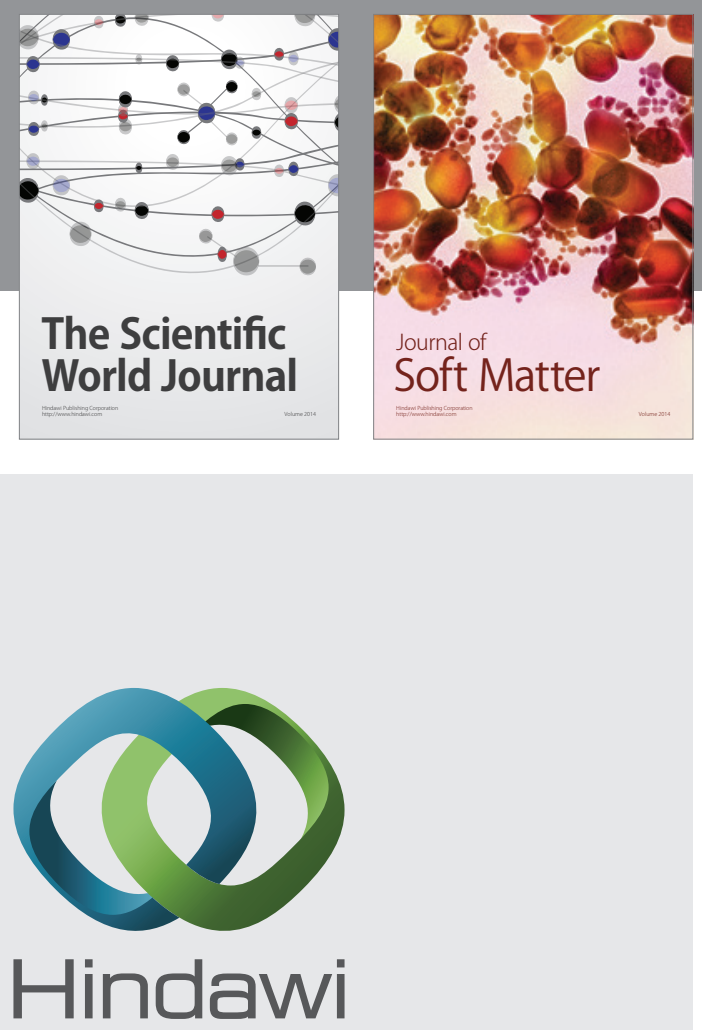

Submit your manuscripts at

http://www.hindawi.com

nternational Journal of

Statistical Mechanics
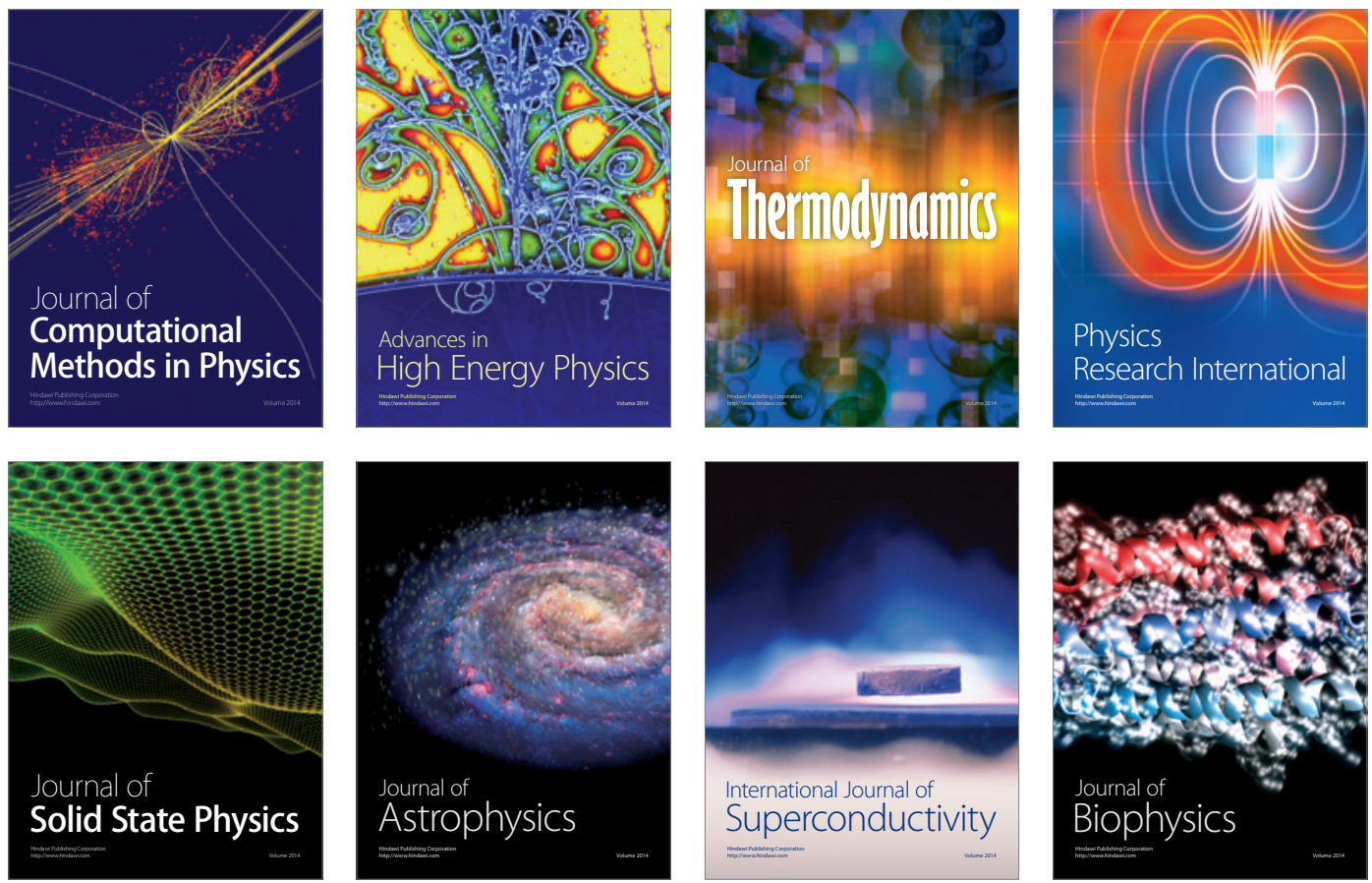
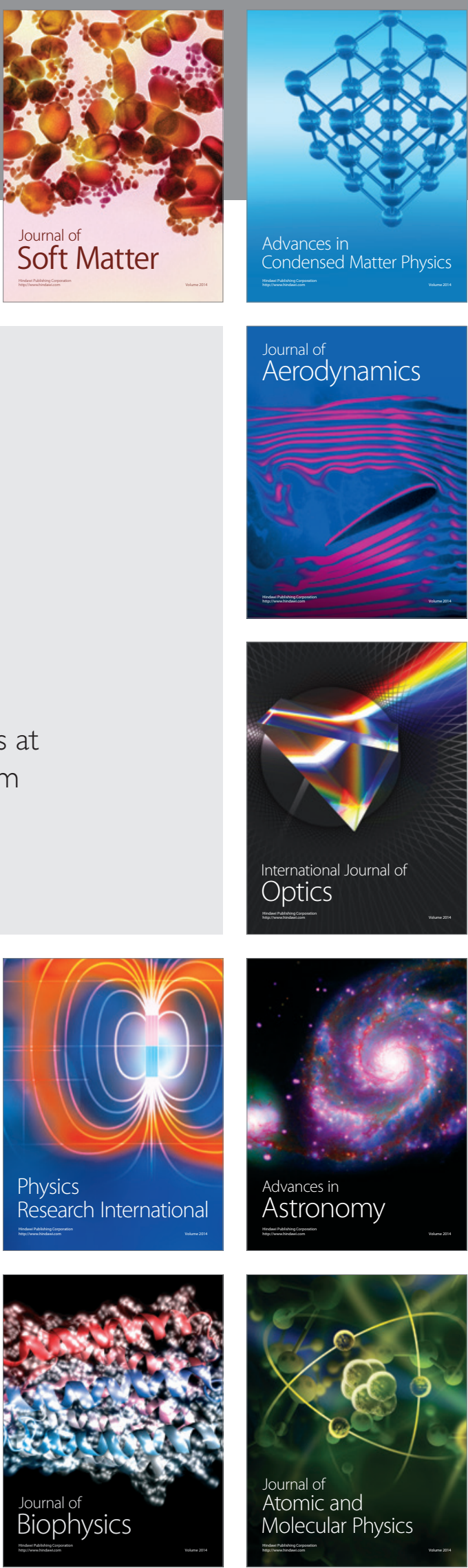